\title{
Large-scale magnetic field in the Monoceros OB 1 east molecular cloud
}

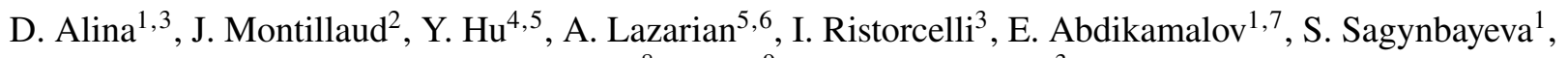 \\ M. Juvela ${ }^{8}$, T. Liu' ${ }^{9}$ and J.-S. Carrière ${ }^{3}$ \\ ${ }^{1}$ Department of Physics, School of Sciences and Humanities, Nazarbayev University, Nur-Sultan 010000, Kazakhstan \\ e-mail: dana. alina@nu.edu.kz \\ 2 Institut UTINAM, UMR 6213, CNRS, Université Bourgogne Franche Comté, France, OSU THETA, 41bis avenue de l'Observatoire, \\ 25000 Besançon, France \\ 3 IRAP, Université de Toulouse CNRS, UPS, CNES, 31400 Toulouse, France \\ ${ }^{4}$ Department of Physics, University of Wisconsin-Madison, Madison, WI 53706, USA \\ ${ }^{5}$ Department of Astronomy, University of Wisconsin-Madison, Madison, WI 53706, USA \\ ${ }^{6}$ Center for Computation Astrophysics, Flatiron Institute, 162 5th Ave, New York, NY 10010, USA \\ ${ }^{7}$ Energetic Cosmos Laboratory, Nazarbayev University, Nur-Sultan 010000, Kazakhstan \\ ${ }^{8}$ Department of Physics, PO Box 64, University of Helsinki, 00014, Helsinki, Finland \\ ${ }^{9}$ Shanghai Astronomical Observatory, Chinese Academy of Sciences, 80 Nandan Road, Shanghai 200030, PR China
}

Received 30 July 2020 / Accepted 11 November 2021

\begin{abstract}
Context. The role of large-scale magnetic fields in the evolution of star-forming regions remains elusive. Its investigation requires the observational characterization of well-constrained molecular clouds. The Monoceros OB 1 molecular cloud is a large complex containing several structures that have been shown to be engaged in an active interaction and to have a rich star formation history. However, the magnetic fields in this region have only been studied on small scales.

Aims. We study the large-scale magnetic field structure and its interplay with the gas dynamics in the Monoceros OB 1 east molecular cloud.

Methods. We combined observations of dust polarized emission from the Planck telescope and CO molecular line emission observations from the Taeduk Radio Astronomy Observatory 14-metre telescope. We calculated the strength of the plane-of-sky magnetic field using a modified Chandrasekhar-Fermi method and estimated the mass-over-flux ratios in different regions of the cloud. We used the comparison of the velocity and intensity gradients of the molecular line observations with the polarimetric observations to trace dynamically active regions.

Results. The molecular complex shows an ordered large-scale plane-of-sky magnetic field structure. In the northern part, it is mostly orientated along the filamentary structures, while the southern part shows at least two regions with distinct magnetic field orientations. Our analysis reveals a shock region in the northern part right between two filamentary clouds that, in previous studies, were suggested to be involved in a collision. The magnetic properties of the north-main and north-eastern filaments suggest that these filaments once formed a single one, and that the magnetic field evolved together with the material and did not undergo major changes during the evolution of the cloud. In the southern part, we find that either the magnetic field guides the accretion of interstellar matter towards the cloud or it is dragged by the matter falling towards the main cloud.

Conclusions. The large-scale magnetic field in the Monoceros OB 1 east molecular cloud is tightly connected to the global structure of the complex. In the northern part, it seems to serve a dynamically important role by possibly providing support against gravity in the direction perpendicular to the field and to the filament. In the southern part, it is probably the most influential factor governing the morphological structure by guiding possible gas inflow. A study of the whole Monoceros OB 1 molecular complex at large scales is necessary to form a global picture of the formation and evolution of the Monoceros OB 1 east cloud and the role of the magnetic field in this process.
\end{abstract}

Key words. ISM: magnetic fields - ISM: clouds - ISM: general

\section{Introduction}

Magnetic fields are one of the key factors regulating the dynamical processes in molecular clouds, alongside gravity and turbulence. Studies of the relative orientation between filamentary molecular clouds and interstellar magnetic fields traced by polarimetric observations of dust emission are one of the main tools in probing how the magnetic field affects the evolution of the interstellar medium (ISM) and the formation of dense structures. Planck Collaboration Int. XXXV (2016) showed that in nearby molecular clouds the relative orientation changes from parallel to perpendicular with increasing column density. The latter effect can be understood on the basis of magnetohydrodynamic (MHD) turbulence properties (Xu et al. 2019) as well as on their inner morphology and evolutionary stage (Liu et al. 2018b; Doi et al. 2020; Soam et al. 2019; Malinen et al. 2016; Alina et al. 2019). Alina et al. (2019) suggested that the highdensity contrast filaments could be those where self-gravity takes over, and the magnetic field turns out to be perpendicular to the overdensities. In particular, MHD simulations reveal that the formation of structures within molecular clouds is highly affected by the magnetic field while the magnetic field strength and 
structure are both affected by the turbulent motions of matter (André et al. 2014; Li et al. 2014; Hennebelle 2013; Federrath 2016).

Polarimetric observations of the interstellar dust allow us to indirectly trace the magnetic field orientation, but it suffers from the signal integration along the line of sight and from the ambiguity coming from the projection of distinct structures onto the plane-of-sky (POS), which, in this case, may appear to be connected. If the former constraint can partly be alleviated for observations in the lines of sight out of the Galactic disk and within the optically thin emission assumption, the latter can only be examined using molecular line data. A recently developed technique based on velocity gradients (Yuen \& Lazarian 2017b; Lazarian \& Yuen 2018b; Hu et al. 2018) provides a promising new way of using spectroscopic data for sampling magnetic fields in molecular clouds (see $\mathrm{Hu}$ et al. 2019c, and references therein).

Relating the information on the dynamics of molecular clouds to their magnetic field structure allows us to better understand how the turbulence, magnetic fields, and gravity regulate dynamical processes in molecular cloud filaments and in the star formation process. To achieve this goal, it is imperative to combine the polarimetric and spectroscopic data. Recently, there has been an increase in synthesized studies. In particular, a combination of Planck and Herschel continuum data and ${ }^{12} \mathrm{CO}$ and ${ }^{13} \mathrm{CO}$ emission data was used by Malinen et al. (2016) to study the L1642 cloud. This allowed the authors to demonstrate a tight connection between the magnetic field structure and the morphology of the cloud and to confirm the connection of striations to the clumps embedded in the cloud. Heyer et al. (2020) also used the Planck polarization data and ${ }^{12} \mathrm{CO}$ and ${ }^{13} \mathrm{CO}$ emission data to study the role of the magnetic field in structuring the Taurus molecular cloud. Their analysis of variations in the relative orientation between the magnetic field and the gradients of surface brightness suggested a presence of local variations of the Alfvénic Mach number at different layers of the cloud. Fissel et al. (2019) compared the orientation of the structures traced by ${ }^{13} \mathrm{CO}$ and $\mathrm{C}^{18} \mathrm{O}$ data with the magnetic field derived from the BLASTPol balloon experiment polarimetric data in the Vela C molecular cloud to infer the density threshold of the transition between parallel and perpendicular relative orientations. It seems necessary to widen the scope of combined analyses of polarimetric and spectroscopic data to connect the magnetic field structure to the dynamical processes in molecular clouds.

The Monoceros OB 1 molecular complex is well suited for such studies. It is located near the Galactic anti-center direction. The eastern part of the complex has a latitude range sufficient to avoid confusion with the background material of the Perseus galactic arm. It also seems to be free of any significant amount of foreground material, according to, for example, the 3D extinction map by Green et al. (2019). Thanks to its intermediate distance of 723 pc (Cantat-Gaudin et al. 2018), it is possible to map the entire complex ( $\sim 60$ pc, i.e., 5 degrees) at millimeter wavelengths with a spatial resolution of the order of the core scales $(\sim 0.15 \mathrm{pc}$, i.e. $\left.45^{\prime \prime}\right)$. Thus, it has been the target of a wealth of observations confirming an active star-forming process. In this study, we focus on the eastern part of the complex. It hosts NGC 2264, a 5 Myr old open cluster containing over 1000 stars, including not only several bright $\mathrm{O}$ and $\mathrm{B}$ stars but also many $\mathrm{T}$ Tauri stars along with signs of ongoing star formation activity such as young stellar objects (YSOs) and dense cores (Park \& Sung 2002; Dahm \& Simon 2005; Chen et al. 2007; Gregorio-Hetem 2003; WolfChase et al. 2003). Furthermore, Rapson et al. (2014) studied the Monoceros OB 1 east cloud using Spitzer data and showed that NGC 2264 and the remainder of the cloud are significantly different regarding the star formation activity. The open cluster is undergoing an active star formation process and its natal gas around the overdensity of YSOs has already been dispersed. The remainder of the cloud has a dispersed population of old stars and signs of recent starburst activity and it is globally quiescent. The dynamics of the filamentary structure of G202.3+2.5, which is located in the northern part of the Monoceros OB 1 east molecular cloud, was extensively studied by Montillaud et al. (2019a,b) using observations of the TRAO (Taeduk Radio Astronomy Observatory) 14-m and IRAM (Institut de Radio Astronomie Millimétrique) 30-m telescopes. They showed that the cloud is likely experiencing a collision of two filaments, and the junction region exhibits signs of intense star formation activity. In contrast, the magnetic field in this region remains poorly known. Dotson et al. (2010) reported polarimetric observations at $350 \mu \mathrm{m}$ toward three targets in Monoceros OB 1. However, those measurements have a limited extent of approximately $2^{\prime}$, focused on selected dense cores.

This paper is aimed to study the Monoceros OB 1 east molecular cloud's large-scale magnetic field and investigate how it is interlaced with the global dynamics. To do so, we use the Planck ${ }^{1}$ telescope polarimetric observations of the interstellar dust emission and the new TRAO 14-metre telescope observations of ${ }^{12} \mathrm{CO}$ and ${ }^{13} \mathrm{CO}(\mathrm{J}=1-0)$ emission. We use the novel technique of comparison of the spectroscopic velocity and intensity gradients with the magnetic field orientation (Yuen \& Lazarian 2017b; Lazarian \& Yuen 2018b) to trace the dynamical processes within MHD turbulence.

The paper is organized as follows: we describe the data and the methods in Sects. 2 and 3, respectively. We present and discuss the results in Sects. 4 and 5. We propose our vision of the interplay between the magnetic fields and the evolution of the cloud in Sect. 6.

\section{Data used}

We combine Planck $353 \mathrm{GHz}$ intensity and polarization data as well as the derived column density map, and the TRAO 14-m telescope observations of the ${ }^{12} \mathrm{CO}$ and ${ }^{13} \mathrm{CO}$ emission that we describe below.

\subsection{Continuum observations and data}

\subsubsection{Planck data}

To trace the magnetic field orientation in the POS, we use the $353 \mathrm{GHz}$ polarized channel data from the Planck PR3 release (Planck Collaboration I 2020). It provides the Stokes $I, Q, U$ maps, and the corresponding noise variances. To increase the signal-to-noise ratio $(\mathrm{S} / \mathrm{N})$, we smooth all maps from nominal angular resolution $\left(5^{\prime}\right)$ up to a resolution of $7^{\prime}$ using a Gaussian kernel. We follow the procedure described in Planck Collaboration Int. XIX (2015), which takes into account the rotation of the local polarization reference frame.

The polarization fraction $(p)$ and polarization angle $(\psi)$ are calculated from the measured Stokes intensity, $I$ and linear polarization parameters $(Q, U)$ as follows:

$p=\frac{\sqrt{Q^{2}+U^{2}}}{I}$

$\psi=0.5 \operatorname{atan}(-U, Q)$,

1 http://www.esa.int/Planck is an ESA mission with participation of NASA and Canada. 
where the two-argument function atan is used to account for the $\pi$-periodicity. The POS magnetic field angle $\psi_{B}$ is obtained via rotation of $\psi$ by $\pi / 2$ :

$\psi_{B}=\psi+\pi / 2$

The Planck data are given in the COSMO convention adopted from Zaldarriaga (1998), this is the reason for which we take the negative value of $U$. Both angles are defined in the range from $-90^{\circ}$ to $90^{\circ}$ in Eq. (2) and are counted positively from Galactic north to east according to the IAU convention. The maps were extracted from all sky maps in HEALPix format provided by the Planck Legacy Archive ${ }^{2}$ and brought to the equatorial coordinates system.

Bias of polarization fraction and angle appears due to the non-linearity of the equations above and due to the presence of noise in the data (Serkowski 1958; Quinn 2012; Montier et al. 2015a), especially at low S/N. We estimate the S/N of the polarization parameters by calculating the classical estimates of the uncertainties (Montier et al. 2015a), which takes into account the full noise variance matrix. This reveals that the uncertainty of the angle in some regions can be high up to $20^{\circ}$ even for reasonable, larger than three, $\mathrm{S} / \mathrm{N}$ of $p$. It is worth noting that the classical estimate fails at low true $\mathrm{S} / \mathrm{N}$ and overestimates the derived S/N (Montier et al. 2015b). Thus, to increase the reliability of the data and of the $\mathrm{S} / \mathrm{N}$ estimation, we compute the Bayesian estimates of $p, \psi$, and the corresponding uncertainties by performing Monte-Carlo simulations to build posterior probability density functions (PDF) as described in Planck Collaboration Int. XIX (2015). In what follows, $p$ and $\psi$ stand for the mean posterior Bayesian estimates of polarization fraction and angle, while $\sigma_{\mathrm{p}}$ and $\sigma_{\psi}$ denote their uncertainties calculated from variances over the PDFs. The resulting $\mathrm{S} / \mathrm{N}$ of $p$ and the uncertainty of $\psi$ are shown in Fig. 1. In this study, we considered only pixels with $\mathrm{S} / \mathrm{N}(p) \geq 2$ and $\sigma_{\psi}<10^{\circ}$. This selection leads to a distribution of $\sigma_{\psi}$ that peaks around $3.5^{\circ}$ (Fig. C.1).

\subsubsection{Column density map}

We also used the Planck data to derive a column density map to address the gravitational stability in Sect. 4.2. We first calculated the colour temperature map based on the Planck 857, 545, and $353 \mathrm{GHz}$ bands and the IRIS $3 \mathrm{THz}$ band, convolved to the same angular resolution $\left(7^{\prime}\right)$. The spectral energy distribution for each pixel is fitted by the modified black-body law $B_{v}(T) v^{\beta}$ using a spectral index $\beta=2$, which corresponds to the value adopted for the Planck Cold Clumps in Planck Collaboration XXVIII (2015). Then the column density map is calculated using the Planck 857 $\mathrm{GHz}$ channel flux density and

$N_{\mathrm{H}_{2}}=\frac{I_{v}}{\mu m_{\mathrm{H}} B_{v}(T) \kappa_{v}}$,

where the dust opacity $\kappa_{v}$ is taken to be $0.1(v / 1 \mathrm{THz})^{\beta} \mathrm{cm}^{2} \mathrm{~g}^{-1}$ according to Beckwith et al. (1990), and $\mu=2.8 \mathrm{amu}$ is the mean molecular weight per $\mathrm{H}_{2}$ molecule. The resulting $N_{\mathrm{H}}$ map is represented in Fig. 2.

\subsection{Molecular line observations and data}

The Monoceros OB 1 molecular complex was observed with the TRAO 14-m telescope (Jeong et al. 2019) as part of the

\footnotetext{
pla.esac.esa.int
}

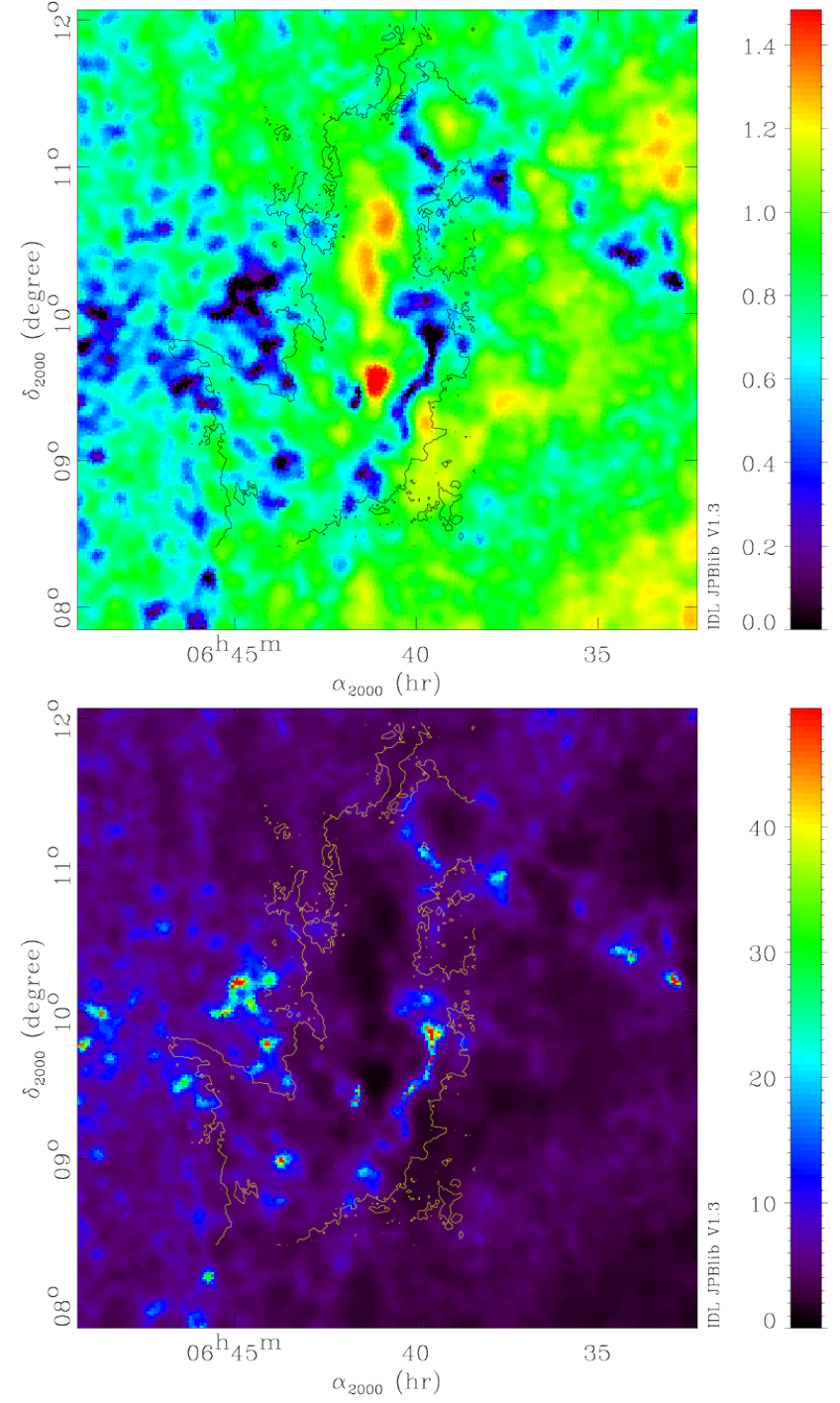

Fig. 1. Assessment of the data quality. Top: $\mathrm{S} / \mathrm{N}$ of the polarization. Colour scale shows $\log _{10}\left(p / \sigma_{\mathrm{p}}\right)$. Bottom: dispersion of the polarization angle $\sigma_{\psi}$ in degrees. Both are estimated using Bayesian analysis of the Planck data. Contours correspond to the value of $2 \mathrm{~K} \mathrm{~km} \mathrm{~s}^{-1}$ of the ${ }^{12} \mathrm{CO}$ integrated intensity, represented in the right panel of Fig. 3.

COMMON large programme (COMplete view of the MONoceros OB 1 molecular complex, P.I.: J. Montillaud), from March till May 2019, and from November 2019 till April 2020. The ${ }^{12} \mathrm{CO}$ and ${ }^{13} \mathrm{CO}(\mathrm{J}=1-0)$ rotational lines at 115.271 and 110.201 $\mathrm{GHz}$, respectively, were detected with the SEQUOIA-TRAO frontend, a $4 \times 4$ multi-beam receiver, with a spectral resolution of $\sim 0.04 \mathrm{~km} \mathrm{~s}^{-1}$ and a beam size of $47^{\prime \prime}$. The observations were conducted in the on-the-fly mode, and the data were reduced with the otftool-TRAO software to produce maps with $20^{\prime \prime}$ cells. After smoothing the spectra to an effective resolution of $0.2 \mathrm{~km} \mathrm{~s}^{-1}$, the achieved sensitivity is $\operatorname{rms}\left(T_{\mathrm{a}}^{*}\right) \approx 0.35 \mathrm{~K}$ and $\approx 0.15 \mathrm{~K}$ for ${ }^{12} \mathrm{CO}$ and ${ }^{13} \mathrm{CO}$, respectively. The dataset is presented in-depth by Montillaud et al. (in prep.). In the present paper, we use the $6 \mathrm{deg}^{2}$ covering the eastern part of the complex, as presented in the right panel of Fig. 3. The Monoceros OB 1 east complex is detected in the velocity range from -3 to $17 \mathrm{~km} \mathrm{~s}^{-1}$, and the analysis of the TRAO data in this paper is restricted to these limits. 


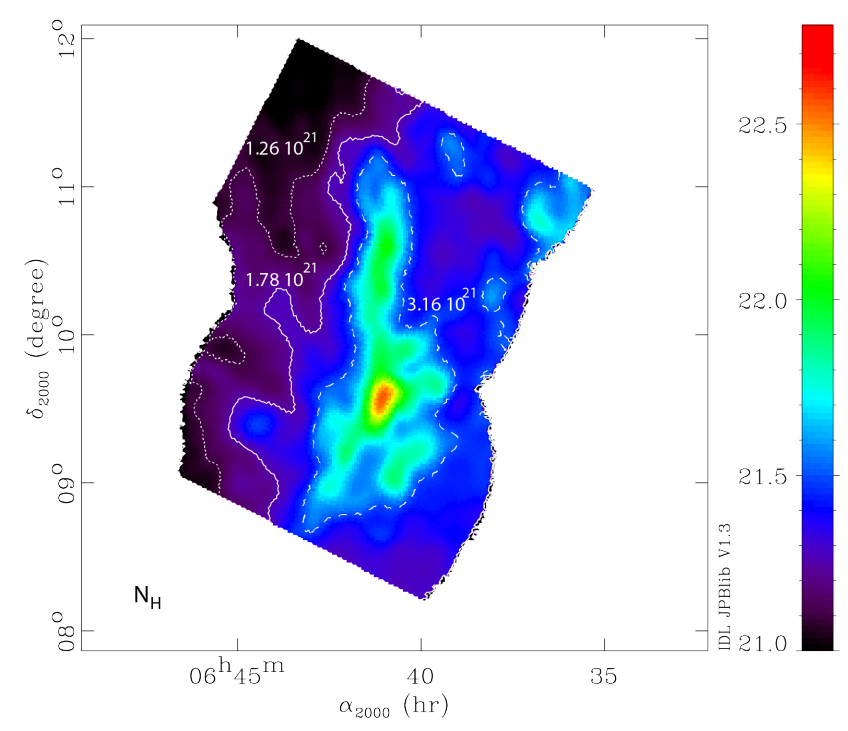

Fig. 2. Column density $\left(N_{\mathrm{H}}\right)$ map derived using the Planck and IRIS data, in the logarithmic scale and in $\mathrm{cm}^{-2}$. The contours correspond to $1.26 \times 10^{21}, 1.78 \times 10^{21}$, and $3.16 \times 10^{21} \mathrm{~cm}^{-2}$.

\section{Methods}

\subsection{Velocity coherent structures}

The TRAO ${ }^{13} \mathrm{CO}$ data were analyzed to identify velocity coherent structures (VCS). We adapted the method presented in Montillaud et al. (2019b), which is, in turn, adapted from Hacar et al. (2013). In short, we start by smoothing each velocity channel with a Gaussian kernel of full width half maximum (FWHM) of $100^{\prime \prime}$, to improve the S/N. Each pixel is then fitted with up to three Gaussian components, and the central velocities of the components peaking at values with $S / N>12$ are used to build a cube of discrete points. A friends-of-friends algorithm is then used to connect the most related points. Two points are considered to be "friends" when they are within a $5 \times 5 \times 5$ box, where pixels are $20^{\prime \prime}$ wide and the channel width is $0.2 \mathrm{~km} \mathrm{~s}^{-1}$. These values are found to provide VCSs small enough to disentangle the main parts of the clouds and large enough to enable an analysis of the magnetic field properties.

\subsection{Determination of the magnetic field strength}

Chandrasekhar \& Fermi (1953) and Davis (1951) led pioneering work on the determination of the magnetic field strength from the dispersion of polarization angles in the Galactic plane (referred to as DCF hereafter). The principle of linking the magnetic field strength to the dispersion of observed angles has been used to study the balance between magnetic and turbulent energies in molecular clouds. Many authors proposed modifications to the method to resolve issues related to observational constraints or the underlying assumptions on the nature of turbulence. From among them, we chose the approach proposed by Houde et al. (2009). It assumes the turbulence isotropic and homogeneous, which is not the case in molecular clouds where gradients of the MHD turbulent velocities are dependent on the magnetic field orientation. However, this method accounts for the effects of beam dilution and integration through the thickness of the cloud, which are the primary sources of overestimation of the magnetic field strength using the DCF method (Crutcher 2012). The method consists in evaluating the dispersion function of polarization angles at different lags, or distance, $l$, expressed by $C=$ $1-\cos \langle\Delta \psi(l)\rangle$, which makes it possible to determine the turbulent $\left(B_{t}\right)$ to ordered $\left(B_{0}\right)$ magnetic field strength ratio through the following equation:

$$
\begin{aligned}
C=1-\langle\cos [\Delta \psi(l)]\rangle & \simeq \sqrt{2 \pi} \frac{\left\langle B_{t}^{2}\right\rangle}{\left\langle B_{0}^{2}\right\rangle}\left[\frac{\delta^{3}}{\left(\delta^{2}+2 R^{2}\right) L}\right] \\
& \times\left[1-\mathrm{e}^{-l^{2} / 2\left(\delta^{2}+2 R^{2}\right)}\right]+a l^{2},
\end{aligned}
$$

where $\delta$ is the turbulent correlation length, $R$ is the telescope beam radius, $L$ is the thickness of the cloud, and $a$ is a parameter defining the large-scale component. The right-hand term of the equation is calculated from the Planck data. For each lag $l$, we divide data into distance bins where the bin width is constant and is equal to $3^{\prime}$. We note that we consider unique pairs of points. For each pair $i, j$, the difference $\Delta \psi_{i j}$ is given through the corresponding Stokes $Q$ and $U$ parameters:

$\Delta \psi_{i j}=0.5 \operatorname{atan}\left(Q_{j} U_{i}-Q_{i} U_{j}, Q_{i} Q_{j}+U_{i} U_{j}\right)$.

The PDF of the uncertainties on $\psi$ in individual pixels are shown in Fig. C. 1 and are generally less than $5^{\circ}$. We estimate the beam radius with $F W H M=7^{\prime}$, and we assume the thickness of the cloud along the LOS in a given region to be equal to the region's width in the POS.

The fit to the dispersion function provides $\left\langle B_{t}^{2}\right\rangle /\left\langle B_{0}^{2}\right\rangle, a$ and $\delta$. The strength of the POS component of the large-scale magnetic field is determined using the DCF formula (Chandrasekhar \& Fermi 1953):

$B_{0}=\sqrt{4 \pi \rho} \sigma(v) \sqrt{\frac{\left\langle B_{0}^{2}\right\rangle}{\left\langle B_{t}^{2}\right\rangle}}$,

where $B_{0}$ is the value we are searching for, while $\left\langle B_{0}^{2}\right\rangle /\left\langle B_{t}^{2}\right\rangle$ is the fitted parameter, $\sigma(v)$ is the LOS velocity dispersion, and $\rho$ is the mass density. We obtain $\sigma(v)$ from the TRAO ${ }^{13} \mathrm{CO}$ emission maps degraded to the same spatial resolution as the Planck data. The density $\rho$ is evaluated for the volume density $n=10^{2} \mathrm{~cm}^{-3}$, which is motivated, first, by $\mathrm{N}_{2} \mathrm{H}^{+}$emission being mostly limited to the cores of the junction region in the IRAM observations of Montillaud et al. (2019b). Second, as we show in Sect. 3.2, the magnetic field orientation derived from the Planck polarization data and the gradient technique applied to the TRAO spectroscopic data shows a better agreement for the ${ }^{12} \mathrm{CO}$ emission. This suggests that we also detect the magnetic field in the extended large-scale structure, from which we derive the velocity coherent structures (described in Sect. 3.1). It is worth noting that in a recent study Evans et al. (2020) argued that most of the ${ }^{13} \mathrm{CO}$ emission in molecular clouds may, in fact, arise from the gas at the density around $10^{2} \mathrm{~cm}^{-3}$. We also make a tentative estimation of the volume density by assuming the depth of the cloud $L$ to be equal to its width (approximate angular widths are taken to be $30^{\prime}, 15^{\prime}, 15^{\prime}$ for the three VCSs described in Sect. 4.2) and using $n\left(\mathrm{H}_{2}\right)=N_{\mathrm{H}_{2}} / L$ as in Liu et al. (2018a). The obtained values range between $\simeq 10^{2}$ and $\simeq 2 \times 10^{2} \mathrm{~cm}^{-3}$ and are on the adopted order of magnitude.

\subsection{Velocity and intensity gradient techniques}

The velocity gradient technique (VGT; González-Casanova \& Lazarian 2017; Yuen \& Lazarian 2017a; Lazarian \& Yuen 2018a) is a new method for tracing magnetic fields. It employs the anisotropic properties of MHD turbulence (Goldreich \& Sridhar 1995) and the theory of turbulent reconnection (Lazarian \& Vishniac 1999), namely: the fact that turbulent eddies are 

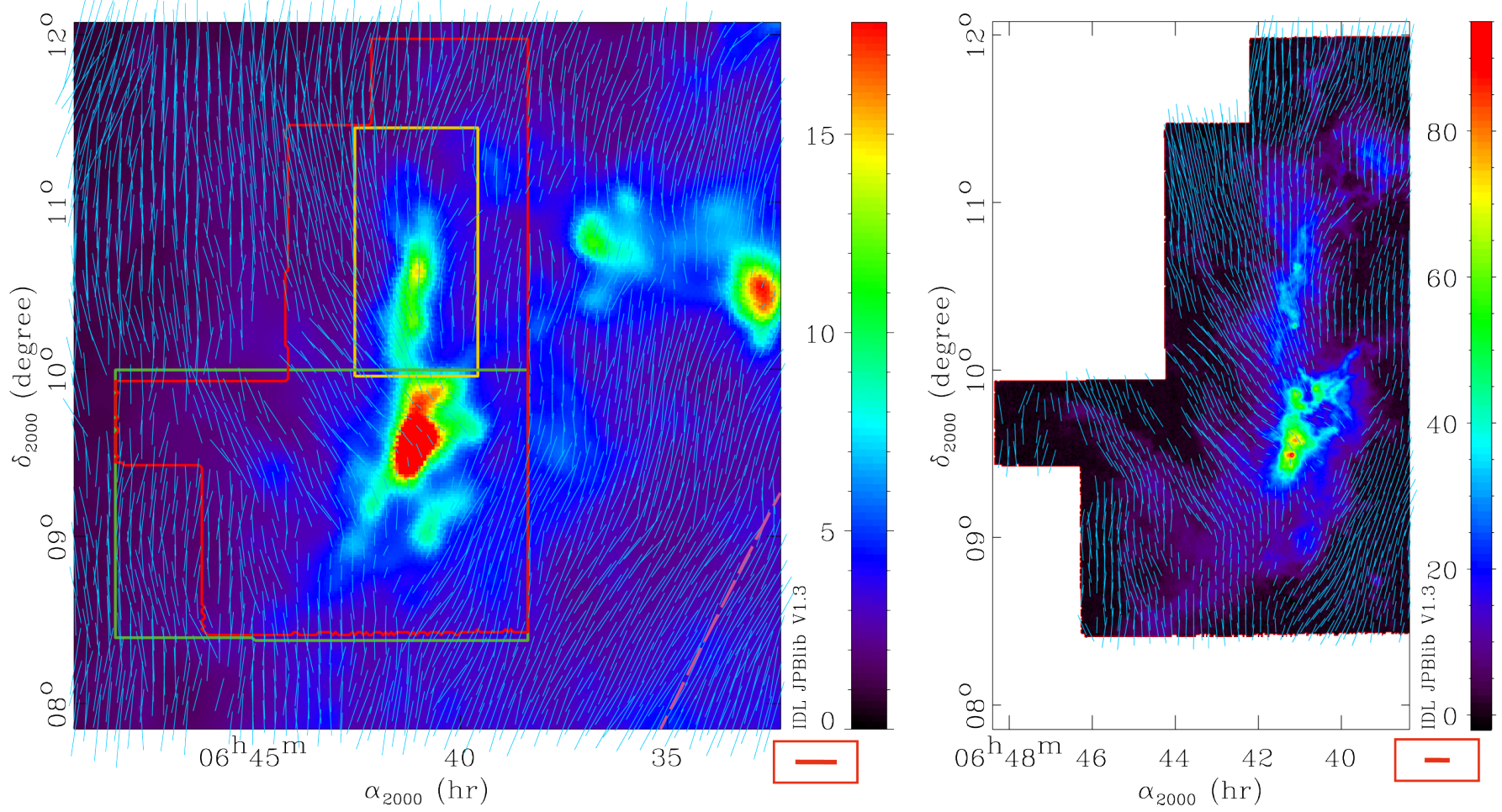

Fig. 3. Monoceros OB 1 east region observed by Planck and TRAO. Left panel: Planck $353 \mathrm{GHz}$ intensity map in $\mathrm{MJy} \mathrm{sr}^{-1}$ at the angular resolution of $7^{\prime}$ with the POS magnetic field orientation overlaid as blue segments. The length of the segments corresponds to polarization fraction, with the reference length of $15^{\prime}$ corresponding to $p=0.05$ (shown in the red box in the bottom right corner). Only data satisfying $\mathrm{S} / \mathrm{N}(p) \geq 2$ and $\sigma_{\psi}<10^{\circ}$ are used for polarization studies. Red, yellow, and green lines show contours of the TRAO map used in this analysis, the northern region (Fig. 5), and the southern region (Fig. 10) respectively. The pink dashed line shows the isolatitude $b_{\mathrm{II}}=0^{\circ}$. Right panel: $\mathrm{TRAO}{ }^{12} \mathrm{CO}(\mathrm{J}=1-0)$ integrated (between $v_{\mathrm{lsr}}=-3$ and $17 \mathrm{~km} \mathrm{~s}^{-1}$ ) $T_{\mathrm{a}}^{*}$ intensity map in $\mathrm{K} \mathrm{km} \mathrm{s}^{-1}$ at angular resolution of $47^{\prime \prime}$ with the POS magnetic field orientation derived from the Planck data overlaid as blue segments. Red contour corresponds to the red contour in the left panel.

elongating along with the local magnetic fields for both subsonic and supersonic cases. For subsonic motions, the velocity and density fluctuations exhibit similar statistical properties so that the velocity gradient (VG) and density gradient (or intensity gradients, IG hereafter) both are perpendicular to the magnetic field. This is the basis upon which the intensity gradient technique $(\mathrm{IGT})^{3}$ was developed (Yuen \& Lazarian 2017a; Hu et al. 2019a).

A molecular cloud is generally supersonic (Zuckerman \& Evans 1974; Padoan et al. 1999; Ballesteros-Paredes et al. 2007). When a shock appears, it breaks the anisotropy relation of the density field. In this case, the IGs would be parallel to the local magnetic fields, rather than perpendicular, in front of shocks. As for the VGs, the relative orientation with respect to the magnetic fields is still perpendicular ( $\mathrm{Hu}$ et al. 2019a). Therefore, the comparison between the VGs and the IGs, can serve as an indication of regions of shock. Another particular situation for VGs and IGs is self-gravity. Hu et al. (2020) numerically demonstrated that the inflow induced by gravitational collapse could flip the orientation of both IGs and VGs by 90 degrees, from

\footnotetext{
3 The IGT should not be confused by the histograms of relative orientation (HRO) proposed by Soler et al. (2013). While both techniques employ intensity gradients, the IGT uses the set of procedures from the VGT to obtain the orientation of the magnetic field. On the contrary, the HRO gets the magnetic field via polarization measurements and compares those with the intensity gradients. A detailed comparison of IGT and HRO is presented in Hu et al. (2019a).
}

perpendicular to parallel to the magnetic field. This phenomenon has been observed in the molecular clouds NGC 1333 and Serpens (Hu et al. 2019c). The comparison of the magnetic field orientations inferred from the interstellar dust polarization measurements with the orientations of VGs can then reveal regions of gravitational collapse and quiescent regions where turbulent motions, thermal pressure, and magnetic support dominate over gravitational energy.

The applicability of the techniques has been observationally demonstrated by making comparisons with Planck $353 \mathrm{GHz}$ polarization and BLASTPol polarization observations (Hu et al. $2019 c, b)$. The notion of tracing the local orientation is important as it means that the gradients can map the detailed structure of magnetic field and not only the mean magnetic field orientation $^{4}$. In the current paper, using the spectroscopic data, this technique allows us to trace the magnetic fields by calculating the IGs and VGs from the integrated intensity map (moment-0) and the velocity centroid map (moment-1), respectively (Yuen \& Lazarian 2017b; Hu et al. 2019a). We briefly describe the calculation procedures in the following.

The gradient calculation is performed by convolving individual 2D moment maps with $3 \times 3$ Sobel kernels $G_{x}$

\footnotetext{
4 The orientation of velocity in turbulent eddies with respect to the local orientation of the magnetic field passing through the eddies follows from the theory of turbulent reconnection (Lazarian \& Vishniac 1999) and is well supported by numerical simulations, e.g., Cho \& Vishniac (2000), Cho et al. (2002).
} 
and $G_{y}$ :

$G_{x}=\left(\begin{array}{lll}-1 & 0 & +1 \\ -2 & 0 & +2 \\ -1 & 0 & +1\end{array}\right), \quad G_{y}=\left(\begin{array}{ccc}-1 & -2 & -1 \\ 0 & 0 & 0 \\ +1 & +2 & +1\end{array}\right)$

as follows:

$\nabla_{x} f(x, y)=G_{x} * f(x, y)$

$\nabla_{y} f(x, y)=G_{y} * f(x, y)$

$\psi_{g}(x, y)=\tan ^{-1}\left(\frac{\nabla_{y} f(x, y)}{\nabla_{x} f(x, y)}\right)$

where $f(x, y)$ represents either moment-0 or moment-1 maps, $\nabla_{x} f(x, y)$ and $\nabla_{y} f(x, y)$ are the $x$ and $y$ components of the gradient respectively, and $*$ denotes the convolution. However, the anisotropy of MHD turbulence concerning the local magnetic field is a statistical concept. The pixelized raw gradient map $\psi_{g}(x, y)$ is not necessarily required to have any relation to the local magnetic field orientation. The perpendicular relative orientation of gradients and magnetic field only appears when the gradient sampling is enough. The statistical sampling procedure utilizes the sub-block averaging method, which is proposed by Yuen \& Lazarian (2017b). The sub-block averaging method first takes all gradient orientations within a sub-block of interest and then plots the corresponding histogram. Because the histogram is close to a Gaussian distribution, the expectation value of the Gaussian distribution reflects the statistically most probable orientation of the gradient. The expectation value of gradients defines the mean gradient orientation for the sub-block and is expected to be perpendicular to the magnetic field.

The velocity channel gradients (VChGs; Lazarian \& Yuen 2018a) are calculated similarly to VGs using the thin velocity channel map $\mathrm{Ch}(x, y)$. Then, $\mathrm{Ch}(x, y)$ is defined as:

$\mathrm{Ch}(x, y)=\int_{v_{0}-\Delta v / 2}^{v_{0}+\Delta v / 2} T_{\mathrm{R}}(x, y, v) \mathrm{d} v$

where $T_{\mathrm{R}}$ is the radiation temperature and $v_{0}$ is the velocity of the averaged emission line maximum. The channel width $\Delta v$ satisfies $\Delta v<\sqrt{\left(\delta v^{2}\right)}$, where $\sqrt{\left(\delta v^{2}\right)}$ is the velocity dispersion. The thin velocity channel maps are used within the assumption that the velocity fluctuations are dominating over density fluctuations due to the velocity caustic effect (Lazarian \& Pogosyan 2000).

The rms noise of the spectroscopic data can, in principle affect the calculation of the gradients. We represent in Fig. C.2 the rms noise of ${ }^{13} \mathrm{CO}$ data which varies from one tile of the mosaic to another between $T \simeq 0.12 \mathrm{~K}$ and $T \simeq 0.18 \mathrm{~K}$. It shows that apart from the tile-to-tile rms variations, no significant structure (e.g., scanning artifacts) in the noise may create spurious dominant gradient orientation in the noise-dominated areas. The linear structures at the junction between adjacent tiles are the strongest noise structures in our dataset and are located in predictable places. We see in the results presented in Sect. 4 that no correlations between the observed gradients and the tile overlaps appear, even in the lowest accepted $\mathrm{S} / \mathrm{N}$ regions. In addition, the sub-block averaging redresses this issue. In this work, we applied IG, VG, and VChG techniques to the TRAO data, with sub-block size of $20 \times 20$ pixels (one pixel is $20^{\prime \prime}$ large) which is the empirical minimum value (Lazarian \& Yuen 2018b); we also smooth the VGs and IGs to 7' resolution of the Planck data. We also tested 30-pixel large box size and observed that the results are stable against averaging. For a fully noise-dominated subregion, the corresponding histogram of the gradients would be a uniform distribution, for which sub-block averaging gives a noise-type output. In the case of a spurious signal, the histogram would exhibit a peak in the given sub-block, and the averaging method minimizes such a contribution. The stability of the results at larger block size suggests this effect is not significant since it is less likely that spurious noise features still dominate at larger scales. To further improve the accuracy of the gradients, we made a selection based upon the error of Gaussian fitting, which is intrinsically based on the noise level of the spectroscopic data. The details of the used thresholds are reported in Sect. 4.3.

\section{Results}

Figure 3 shows the $353 \mathrm{GHz}$ dust emission map and the ${ }^{12} \mathrm{CO}$ integrated intensity map of the Monoceros OB 1 east molecular cloud. It spans over more than $25 \mathrm{pc}$ in the south-north direction and has a complex morphology. We divide the cloud into two sub-regions: the northern part, which has a clear filamentary shape, and the southern part that includes the NGC 2264 open cluster. Its location corresponds to the red color area in the Planck intensity map. In the left panel of Fig. 3 the northern and southern parts are delimited with yellow and green boxes, respectively. As we shall see, the large-scale magnetic field in the two parts has different properties regarding its structure and its dynamical role.

We observe that the Stokes I map (Fig. 3, left panel), the column density map (Fig. 2) and the integrated CO gas emission map at the velocities of the studied cloud (Fig. 3, right panel) trace the same structure, at column densities larger than $\sim 1.8 \times 10^{21} \mathrm{~cm}^{-2}$. This threshold is used to define the regions where the background polarized signal can be neglected with respect to the polarization coming from the Monoceros OB 1 east cloud.

In what follows, we adopt the nomenclature introduced by Montillaud et al. (2019b) for the sub-structures in the northern part. There, the north-main filament is located in the lower part, connected to two upper filaments that extend slightly to east and west. Henceforth, these are referred to as the north-eastern and north-western, respectively, and the three filaments are connected at the junction region. The schematic representation of these structures is shown in Fig. 4.

\subsection{Plane-of-sky magnetic field structure}

The left panel of Fig. 3 shows the Planck intensity map and the POS magnetic field orientation represented by blue segments. The length of the segments corresponds to the value of $p$ in a given pixel. The Monoceros OB 1 east molecular cloud is located around two degrees above the Galactic plane, and we observe the uniformly orientated magnetic field in the southwestern part of the map, which is globally aligned with the Galactic plane, with the $b_{\mathrm{II}}=0^{\circ}$ latitude shown by the pink dashed line. Moving from the Galactic plane towards the center of the map, the POS magnetic field orientation changes gradually with increasing intensity, then shifts by 90 degrees from the SE-NW (south-east-north-west) to the SW-NE (south-westnorth-east) orientation at the western border of the main part of the cloud. The SW-NE orientation prevails throughout the densest part. The low-intensity eastern part of the map also shows a uniform orientation of the magnetic field, orientated north, that changes in the brighter region. We observe that the northern and southern parts of the cloud show different POS magnetic field structures. 


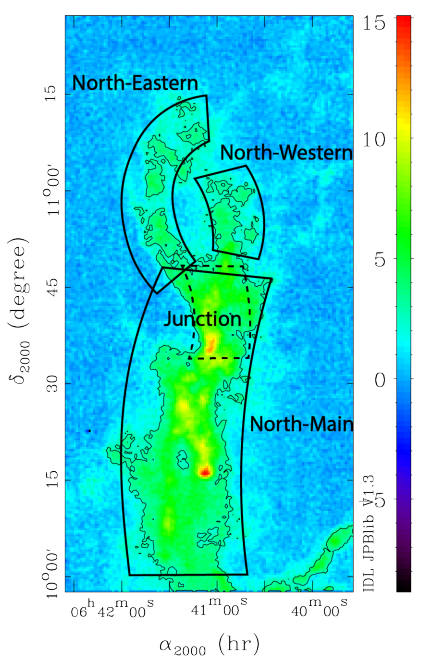

Fig. 4. TRAO ${ }^{13} \mathrm{CO}$ integrated (between $v_{\mathrm{lsr}}=-3$ and $17 \mathrm{~km} \mathrm{~s}^{-1}$ ) $T_{\mathrm{a}}^{*}$ emission map of the northern part of the cloud in $\mathrm{K} \mathrm{km} \mathrm{s}^{-1}$ with the schematic representation of the sub-structures. Contours are taken at $5 \mathrm{~K} \mathrm{~km} \mathrm{~s}^{-1}$.
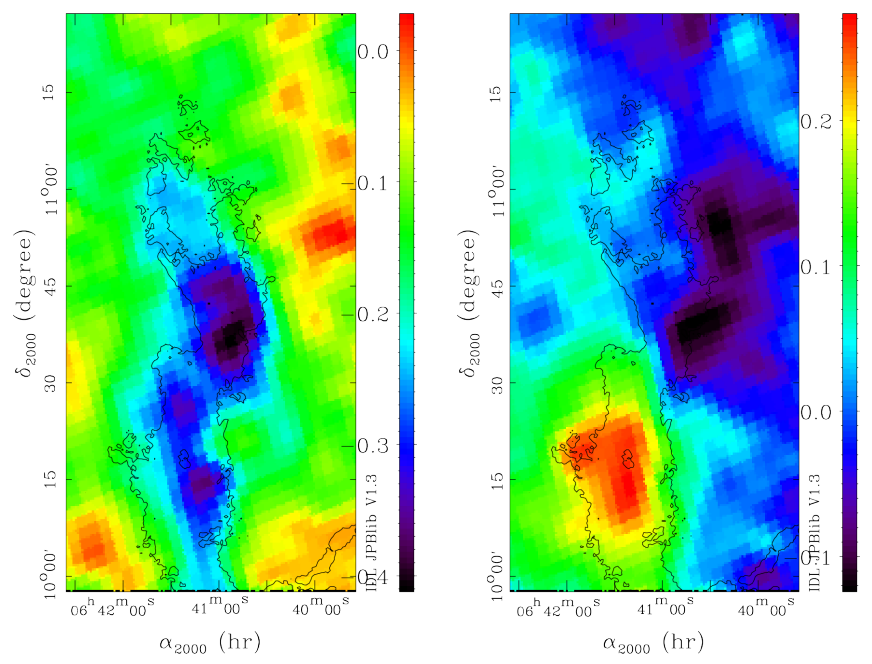

Fig. 5. Planck Stokes $Q$ (left panel) and $U$ (right panel) maps of the northern part of Monoceros OB 1 east, shown in yellow box in Fig. 3 . Contours correspond to the TRAO ${ }^{13} \mathrm{CO}$ emission, as shown in Fig. 4.

The northern part of the cloud shows a large-scale magnetic field mainly orientated in the south-north direction. In order to analyze the variations throughout the cloud, we show in Fig. 5 the maps of polarized linear intensities in that region, $Q$ and $U$, used for the calculation of $\psi_{B}$ (Eq. (3)), so that their relative variations partially reflect variations of $\psi_{B}$. The north-main filament and the north-eastern filament, which are proposed by Montillaud et al. (2019b) to form one structure, are clearly detected in $Q$ as a strong negative signal in a primarily uniform background with the maximum (in absolute value) in the junction region. The $U$ parameter shows a gradient in the direction from south-east to north-west. Remarkably, the north-eastern and north-western filaments have opposite $U$ polarities (positive and negative respectively). This means the magnetic field orientation is systematically directed north with an inclination to the east and west, respectively. This is also seen when inspecting the map of the magnetic field angles in Fig. 3 and when considering the filamentary structures detected in the Planck column density map using RHT (see details in Appendix A). The mean magnetic field angles are around $3^{\circ}$ and $-2.6^{\circ}$ with the mean dispersion of $5.4^{\circ}$ and $5.3^{\circ}$ in the north-eastern and north-western filaments, respectively. The decrease in $Q$ lies between the junction region and the north-main filament, and $U$ changes its sign at the southernmost end of the junction region. This suggests that the two structures have distinct polarization properties.

The southern part of Monoceros OB 1 east, shown in the green box in Fig. 3, has a different magnetic field structure. In particular, at the eastern border, we observe a region with a distinct uniform magnetic field orientated roughly perpendicular to the Galactic plane, extending from low-intensity regions towards the brightest part, which includes NGC 2264. We note that the brightest part seems to be perpendicular to this uniform magnetic field. The southernmost end of the region shows a global N-S orientation of the POS magnetic field inferred from the Planck data, and the two meet in the brightest part of the cloud (see the right panel of Fig. 3). The global SW-NE orientation is also detected in the southernmost western corner of the northern part.

\subsection{Strength of the magnetic field and the effect on filaments stability}

We estimate the POS magnetic field strength according to Sect. 3.2 in the north-eastern, north-western, and north-main filaments, in particular, in VCSs that are identified in ${ }^{13} \mathrm{CO}$ emission (described in Sect. 3.1). This allows us to constrain the spatial extent of the regions and the associated velocity profiles and their dispersions. We require each VCS to contain at least 100 pixels (after projection onto the Planck grid). The northmain filament contains several VCSs in the junction region, and we do not consider that part of the filament to avoid confusion. We also note that the VCS corresponding to the north-eastern filament covers mainly the northern part of the filament. The contours of the VCS and the velocity dispersion map are represented in Fig. 6. We can see that the three VCS do not spatially overlap, and because each of the structures accounts for most of the observed emission, the use of the Planck polarization data is justified.

To estimate the ratio of turbulent to ordered magnetic field strengths, we computed the angular dispersion function $C$ and the corresponding fits as described in Sect. 3.2. The results are shown in Fig. 7. The uncertainties on the measurement of the Stokes $Q$ and $U$ in the Planck data propagate non-linearly to $C$. We used Monte-Carlo simulations that consider the noise variances of $Q$ and $U$ and their co-variance noise. The range of the uncertainties, within $95 \%$ confidence interval, is indicated in dark grey in Fig. 7, except for the north-main region where the uncertainties are minor and are comparable to the symbol size. The areas of the VCS have elongated shapes, and the largest distances have a smaller number of pixels pairs. We represent the largest circles inscribed in each VCS in Fig. 6 and report the corresponding diameters in Fig. 7. This corresponds to the lags for which the determination of pixel pairs is isotropic. We also assessed the uncertainty due to the decreasing number of pixel pairs with increasing lag $l$ for each VCS using MonteCarlo simulations of random angles and report them as gray shaded areas in Fig. 7. The fit was applied to intermediate lags because, at low separations between data points, the assumption of the Gaussian turbulent auto-correlation function is not relevant (Houde et al. 2009), while at large separations, one might be tracing uncorrelated components. The resulting estimates of the POS magnetic field strength are reported in Table 1. The uncertainties are estimated from, first, the standard error propagation between the data and the fit, and second, from the 

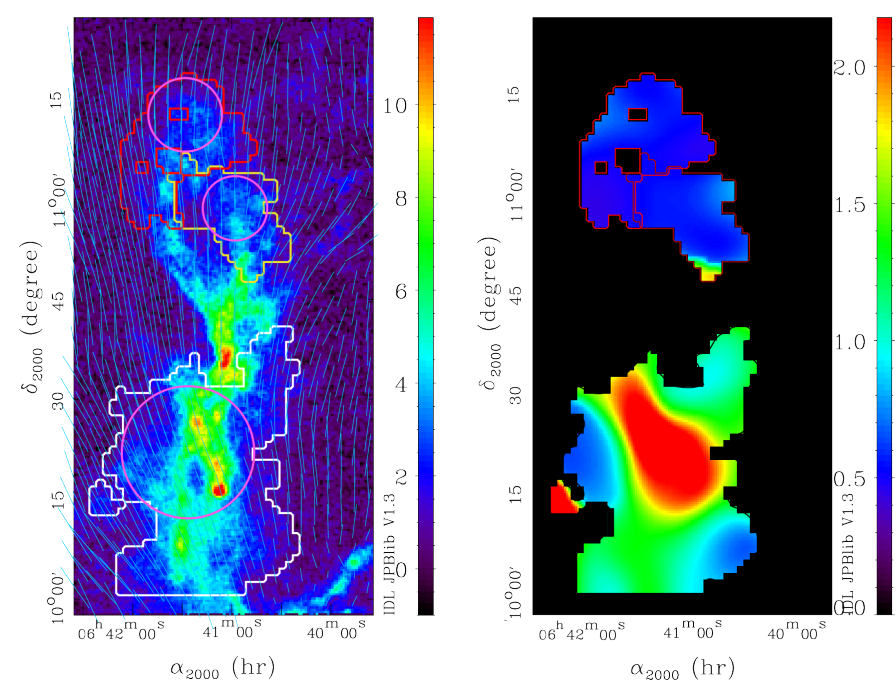

Fig. 6. Northern part of the cloud where the magnetic field strength was estimated. Left panel: location of the north-main (white contour), north-western (yellow contour), and north-eastern (red contour) velocity coherent structures overlaid on the TRAO ${ }^{13} \mathrm{CO}$ integrated intensity map, in $\mathrm{K} \mathrm{km} \mathrm{s}^{-1}$. Segments represent the POS magnetic field orientation derived from the Planck data, the length of the segments corresponds to the polarization fraction, with the reference length of $10^{\prime}$ corresponding to $p=0.05$. Right panel: velocity dispersion in the three subregions from the ${ }^{13} \mathrm{CO}$ emission TRAO data, smoothed to $7^{\prime}$ resolution, in $\mathrm{km} \mathrm{s}^{-1}$. Purple circles correspond to the largest circles inscribed in the area of each VCS.

Monte-Carlo simulations. We find that the strength is around $17 \mu \mathrm{G}, 5 \mu \mathrm{G}$, and $6 \mu \mathrm{G}$ in the north-main, north-western, and north-eastern regions, respectively, or globally on the order of $10 \mu \mathrm{G}$. This value is in agreement with the global tendency in molecular clouds at the observed densities (Crutcher et al. 2010). A determination of the magnetic field strength using the DCF method is generally subject to several assumptions, as well as the approach adopted here. Supposing that errors are similar in different regions, a relative analysis of the results allows us to conclude that the north-western region has a larger turbulent component $\left(\left\langle B_{t}^{2}\right\rangle /\left\langle B_{0}^{2}\right\rangle \simeq 0.67\right.$ compared to $\simeq 0.14$ and 0.11$)$ with the large-scale magnetic field strength two times weaker than in north-main filament. The values of $B_{0, \mathrm{POS}}$ reported here are tentative, and a possible range will be discussed in Sect. 5. As far as we know, there are no Zeeman lines splitting observations in G202.3+2.5 (the northern part). In the NGC 2264 protocluster located half-a-degree south to the north-main region, the Zeeman lines splitting observations were studied by Maury et al. (2012). However, in that region, we observe a different geometry characterising the POS magnetic field. The magnetic field component projected onto the line of sight (LOS) was estimated to be $600 \mu \mathrm{G}$ at most, using IRAM 30-m observations of CN(1-0) emission line at a resolution of $23^{\prime \prime}$. The Zeeman measurement with IRAM traces the small-scale magnetic field strength in the NGC 2264, while the Planck + DCF-derived magnetic field strength applies to the large-scale magnetic field of G202.3+2.5; thus, they cannot be directly compared.

We compare our results with the results derived using a method that does not account for the beam dilution. Hildebrand et al. (2009) proposed using the two-point angular structure function within the assumption of the independence between the large-scale ordered and turbulent components that introduces no assumptions on the geometry of the large-scale field. The
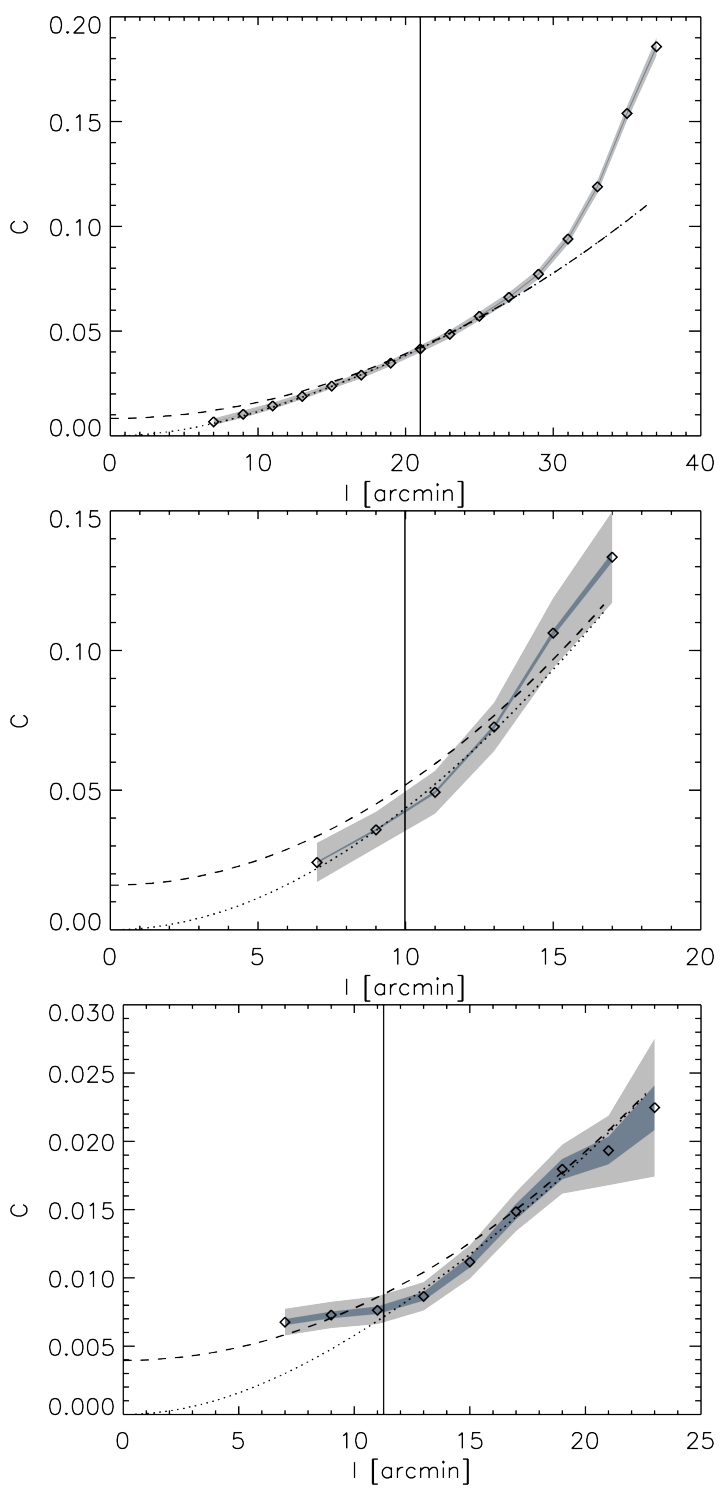

Fig. 7. Angular dispersion functions $C(C=1-\cos (\langle\Delta \psi(l)\rangle))$ and the corresponding fits to the data using Eq. (5) (dotted curves) for the north Main, north western, and north eastern regions from top to bottom respectively. The dashed curves show the integrated turbulent component obtained from Eq. (5) without the exponential term. The grey area shows the error estimated using Monte-Carlo simulations that take into account the noise variances of the data and the dark gray area shows the uncertainty due to the number of available pixel pairs for each lag $l$. The vertical lines show the diameters of the purple circles from Fig. 6.

Table 1. Results of the fit parameter $\left\langle B_{t}^{2}\right\rangle /\left\langle B_{0}^{2}\right\rangle$ from Eq. (5)

\begin{tabular}{ccccc}
\hline \hline Region & $\frac{\left\langle B_{t}^{2}\right\rangle}{\left\langle B_{0}^{2}\right\rangle}$ & $\begin{array}{c}B_{0, \mathrm{POS}} \\
(\mu \mathrm{G})\end{array}$ & $\begin{array}{c}B_{0, \mathrm{POS}}{ }^{(1)} \\
(\mu \mathrm{G})\end{array}$ & $\lambda$ \\
\hline North-main & 0.14 & $16.0 \pm 2.5$ & $385 \pm 17$ & $0.95 \pm 0.41$ \\
North-western & 0.67 & $5.1 \pm 0.9$ & $362 \pm 14$ & $2.55 \pm 0.57$ \\
North-eastern & 0.11 & $6.5 \pm 1.1$ & $85 \pm 2$ & $1.40 \pm 0.38$ \\
\hline
\end{tabular}

Notes. The magnetic field strength $B_{0, \mathrm{POS}}$ is derived using Eq. (5), $B_{0, \mathrm{POS}}^{1}$ is derived using the angular structure function (see Appendix B). $\lambda$ is the estimated mass-to-magnetic flux ratio parameter calculated using Eq. (10). Results are shown in the three detected VCSs in the northern part. ${ }^{(1)}$ calculated according to the method of Hildebrand et al. (2009). 
details of the calculations are reported in Appendix B, and the magnetic field strength estimation is reported in the fourth column of Table 1. This method yields values that are about two orders of magnitude larger for the ordered component's strength and proves the necessity of accounting for the beam dilution to any data. It was also noted by Planck Collaboration Int. XXXV (2016) that the method proposed by Hildebrand et al. (2009) should be applied with caution to the Planck data.

The magnetic field may support the filamentary clouds against gravitational fragmentation. To quantify this, we evaluated the ratio $\lambda$ between the actual and critical mass-to-magnetic flux ratios $M / \Phi$ according to Crutcher (2004):

$\lambda=7.6 \times 10^{-21} N_{\mathrm{H}_{2}} / B_{\mathrm{tot}}$,

where $B_{\text {tot }}$ is the total magnetic field strength in $\mu \mathrm{G}$ and $N\left(\mathrm{H}_{2}\right)$ is the column density of molecular hydrogen in molecules per $\mathrm{cm}^{2}$. We then determine the total magnetic field strength out of our estimation of $B_{\text {POS }}$ : Heiles \& Crutcher (2005) argued that statistically $B_{\mathrm{POS}}$ accounts for 0.79 of $B_{\text {tot }}$ on average, and we adopt this value. If $\lambda<1$, the cloud is subcritical, which means that the cloud is not prone to collapse, and $\lambda>1$, the gravitational energy is larger than the support provided by the magnetic field. We average the column density data in the three VCS regions and obtain $\lambda=0.95 \pm 0.41,2.55 \pm 0.57$, and $1.40 \pm 0.38$ for the north-main, north-western, and north-eastern regions respectively. These regions are mostly supercritical, where the magnetic field alone does not provide sufficient support against collapse. As we show in Sect. 4.3, the VGs do not show significant signs of collapse. Thus, turbulence may be the most likely factor providing support in this instance.

\subsection{Coupling between magnetic field structure and the cloud's dynamics}

According to $\mathrm{Hu}$ et al. (2020), the relative orientation between the magnetic field as inferred from polarimetric dust observations and the velocity and intensity gradients of the gas emission can be used as an indicator of undergoing dynamical processes: (i) if both the VGs and IGs are perpendicular to the magnetic field, this is an indication that the gravity is not a dominant factor in the region; (ii) if both the VGs and IGs are parallel to the magnetic field, this is an indication of a gravitational collapse; (iii) if the VGs are perpendicular to both the magnetic field and the IGs, this is an indication of a shock.

We computed the IGs, VGs, and VChGs in the two parts of the cloud and compared the derived directions between them and to the direction of the POS magnetic field. The uncertainty on the determination of the gradient angles can come from the systematic error in the map and from the sub-block averaging as described in Sect. 3.3. In the sub-block averaging process, only the statistically crucial angle, which corresponds to the peak of the Gaussian fit to the histogram over the considered pixels, is considered, and the uncertainty of the sub-block averaging is taken as the error of the Gaussian fitting at the two-sigma level. Figure 8 shows the histograms of the gradient uncertainties. The PDFs peak between 5 and 10 degrees. We only considered those gradients with uncertainties lower than $30^{\circ}$. Figure 9 illustrates that the data in the northern region has a sufficient $\mathrm{S} / \mathrm{N}$ so that the gradients are defined at every data point. In contrast, Fig. 10 illustrates that in faint regions there are no gradients that satisfy our selection criteria. We set thresholds on the considered area at the ${ }^{12} \mathrm{CO}$ integrated intensity level of $3 \mathrm{~K} \mathrm{~km} \mathrm{~s}^{-1}$ in the southern part and $6 \mathrm{~K} \mathrm{~km} \mathrm{~s}^{-1}$ in the northern part. This
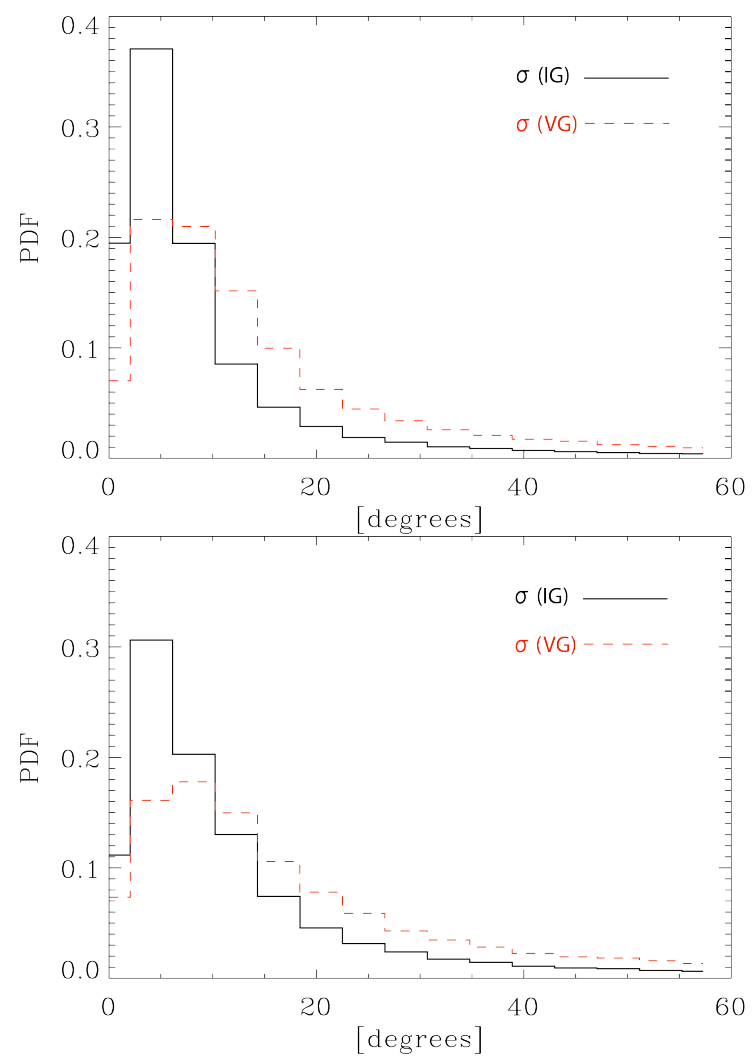

Fig. 8. Uncertainties of the IGs (black plain curve) and VGs (red dashed curve) orientations in the Monoceros OB 1 east cloud. Top: ${ }^{12} \mathrm{CO}$, bottom: ${ }^{13} \mathrm{CO}$.

globally corresponds to column densities above $\sim 1.8 \times 10^{21}$ $\mathrm{cm}^{-2}$ as discussed earlier. Comparison between spectroscopic and polarimetric results is performed above these thresholds.

\subsubsection{Northern part}

We represent in the panel a of Fig. 9 the POS magnetic field traced by the interstellar dust polarized emission, and the VGs and IGs rotated by $90^{\circ}$, calculated on the ${ }^{12} \mathrm{CO}$ TRAO data in the northern part of Mon OB 1 east association, for illustration purpose. We compute the angle differences between magnetic fields and VGs and IGs and represent the PDFs in Fig. 11. Globally, the PDFs peak at $90^{\circ}$, and a $30^{\circ}$ range from the peak corresponds to more than $1 \sigma$ level. In what follows, we adopt this margin as a confident one. In panels b-e of Fig. 9, we overlay the three dynamical cases on the grey scale image of the ${ }^{13} \mathrm{CO}$ integrated intensity map. There, the quiescent regions (case i) are represented as blue shaded areas, the shock regions (case iii) are represented as yellow shaded areas, and the sites of possible collapse (case ii) are shown as red shaded areas. We identify two main types of dynamical regions in the northern part: the "quiescent" regions where the IGs and VGs are both perpendicular to the magnetic field orientation within $30^{\circ}$ (case i) and the candidate "shock" regions where the VGs are perpendicular to, and the IGs are parallel to the magnetic field within $30^{\circ}$ (case iii). The former means that the magnetic field orientation derived from the gradient orientations (obtained by rotation of $90^{\circ}$ ) would give the same orientation of the magnetic field as the one derived from dust polarization observations. In the north-main and 
a) ${ }^{12} \mathrm{CO}$, smoothed

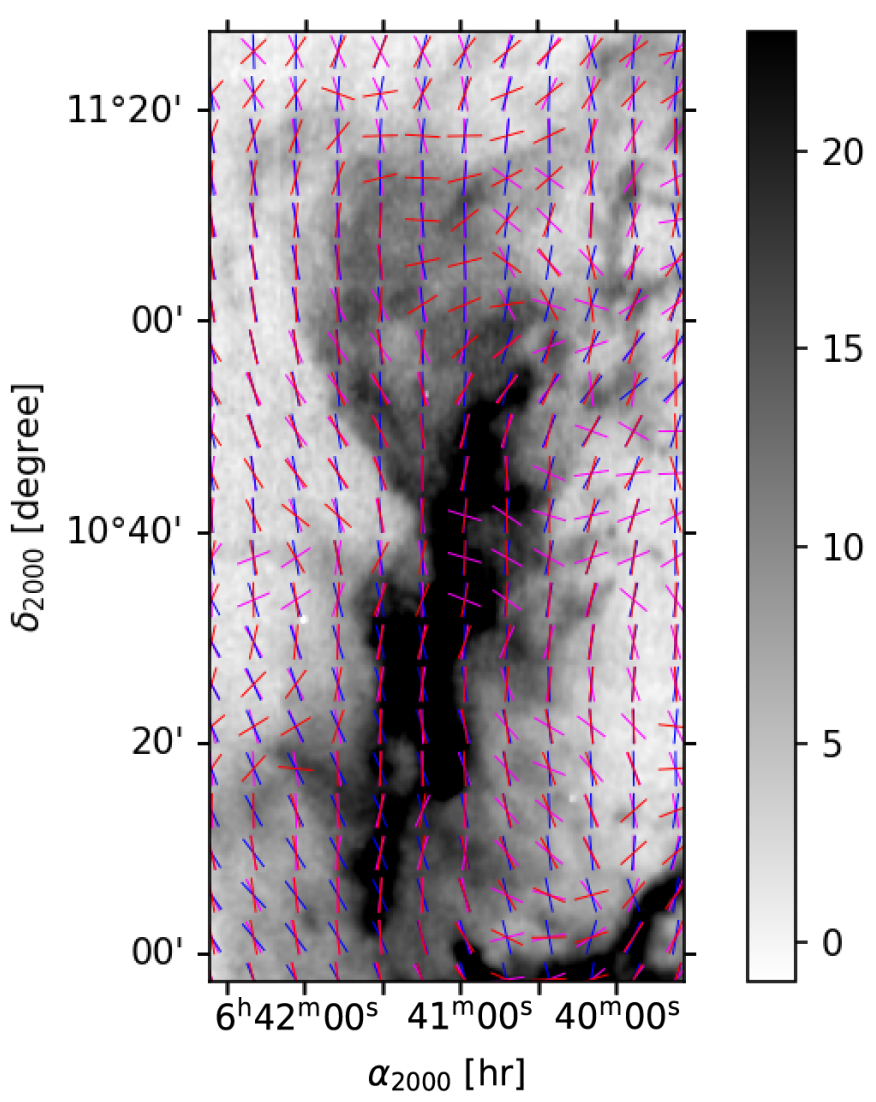

b) ${ }^{12} \mathrm{CO}$, smoothed

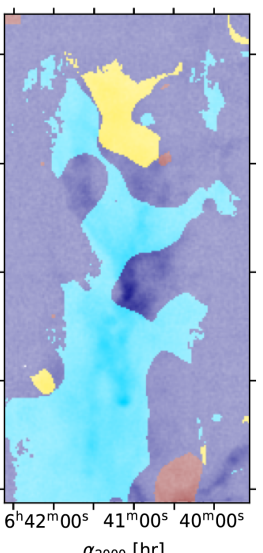

d) ${ }^{12} \mathrm{CO}$, unsmoothed

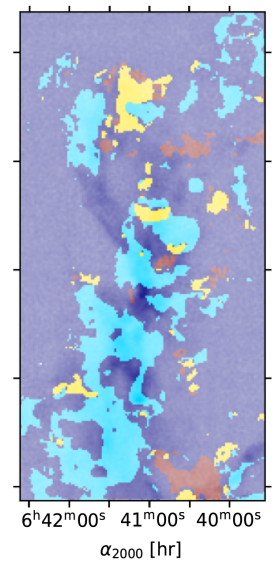

c) ${ }^{13} \mathrm{CO}$, smoothed

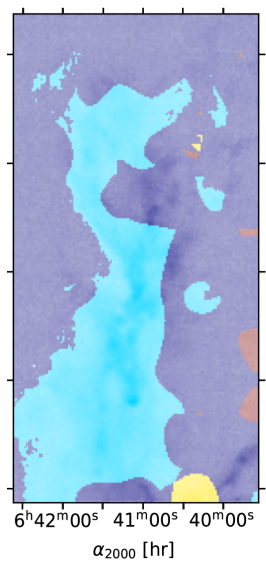

e) ${ }^{13} \mathrm{CO}$, unsmoothed

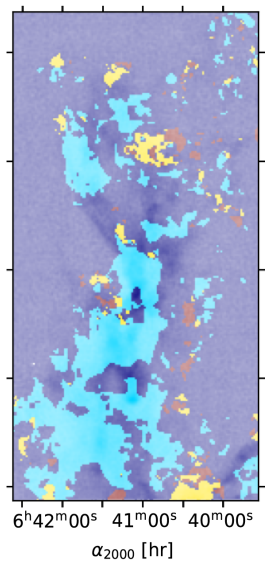

Fig. 9. Northern part of Mon OB 1 east (corresponding to the yellow rectangle in Fig. 3) with the POS magnetic field orientation (blue segments), the orientations of the rotated velocity gradients (magenta segments), and intensity gradients (red segments) overlaid on the TRAO ${ }^{12} \mathrm{CO}$ integrated intensity map (between $v_{\mathrm{lsr}}=-3$ to $17 \mathrm{~km} \mathrm{~s}^{-1}$ ) shown in panel $a$. In panels $b-e$, we show the same region. The blue shaded pattern corresponds to the regions where gravity is not dominant (case i), the red shaded pattern corresponds to the candidate collapse (case ii), the yellow shaded pattern corresponds to the candidate shock regions (case iii), overlaid on the ${ }^{13} \mathrm{CO}$ integrated intensity grey scale map: $(b)$ based on ${ }^{12} \mathrm{CO}$ TRAO data smoothed to $7^{\prime}$ resolution, $(c)$ based on ${ }^{13} \mathrm{CO}$ TRAO data smoothed to $7^{\prime}$ resolution, $(d)$ based on ${ }^{12} \mathrm{CO}$ TRAO data at the resolution of $47^{\prime \prime}$, (e) based on ${ }^{13} \mathrm{CO}$ TRAO data at the resolution of $47^{\prime \prime}$.

north-eastern filaments the ${ }^{12} \mathrm{CO}$ and ${ }^{13} \mathrm{CO}$ intensity and velocity gradients both agree with polarimetric data in estimating the magnetic field orientation. It means that, first, there is apparently no source of foreground contamination in the polarized emission map, and both polarimetric and spectroscopic data trace the same media. Second, the turbulent motions in the gas and the magnetic field support are more significant than the gravitational forces (case i), as expected from the theory on MHD turbulence forming the basis of the gradient technique used in this study. Interestingly, the comparison of the ${ }^{12} \mathrm{CO}$ VGs and IGs with the dust-derived POS magnetic field in panel $b$ traces a shock inbetween the north-eastern and north-western filaments. At the same time, we observe no such dynamics in ${ }^{13} \mathrm{CO}$ (panel c).

It is worth noting that the VG and IG maps were smoothed to the Planck resolution in order to facilitate a comparison with the polarimetric data. However, these maps bring information on the magnetic field structure and the gas dynamics at higher resolution. We oversampled the Planck map and represent in the last two panels $d$ and e of Fig. 9, the dynamically active regions identified using the unsmoothed map of ${ }^{12} \mathrm{CO}$ and ${ }^{13} \mathrm{CO}$. The corresponding histograms are reported in Appendix C, Fig. C.3. As expected, the shock is also observed when using unsmoothed ${ }^{12} \mathrm{CO}$ gradients between the two northern filaments (panel $\mathrm{d}$ ).
Moreover, there are signs of collapse around the shock region and in the north-western filament. In denser parts, traced by ${ }^{13} \mathrm{CO}$, the comparison between the unsmoothed gradients and the oversampled Planck magnetic fields reveals a small shock region between the two filaments, which disappears if the smoothing is applied. In the northern part of the junction region, in ${ }^{13} \mathrm{CO}$ (panel e), we do not observe any noticeable trend. However, if the smoothing is applied, the magnetic field orientation derived from the spectroscopic data is coherent with the magnetic field orientation derived from the polarization data in this region. This can be due to the confusion of multiple sub-structures of Monoceros OB 1 along the LOS (Montillaud et al. 2019b). The coherence between magnetic field orientation derived using polarimetric and spectroscopic data is also lost at the southernmost end of the junction region, where a signature of merging of the northeastern and north-main filaments was detected using the IRAM 30-m observations (Montillaud et al. 2019b). A comparison with higher resolution polarimetric data is necessary in order to analyze the magnetic field in this region. The velocity channel gradients (VChGs) show a better agreement with the Planck data in the determination of the magnetic field orientation: their PDFs show a narrower peak toward $90^{\circ}$, while the VG's PDFs are flatter, especially for the ${ }^{13} \mathrm{CO}$ data (bottom panel of 

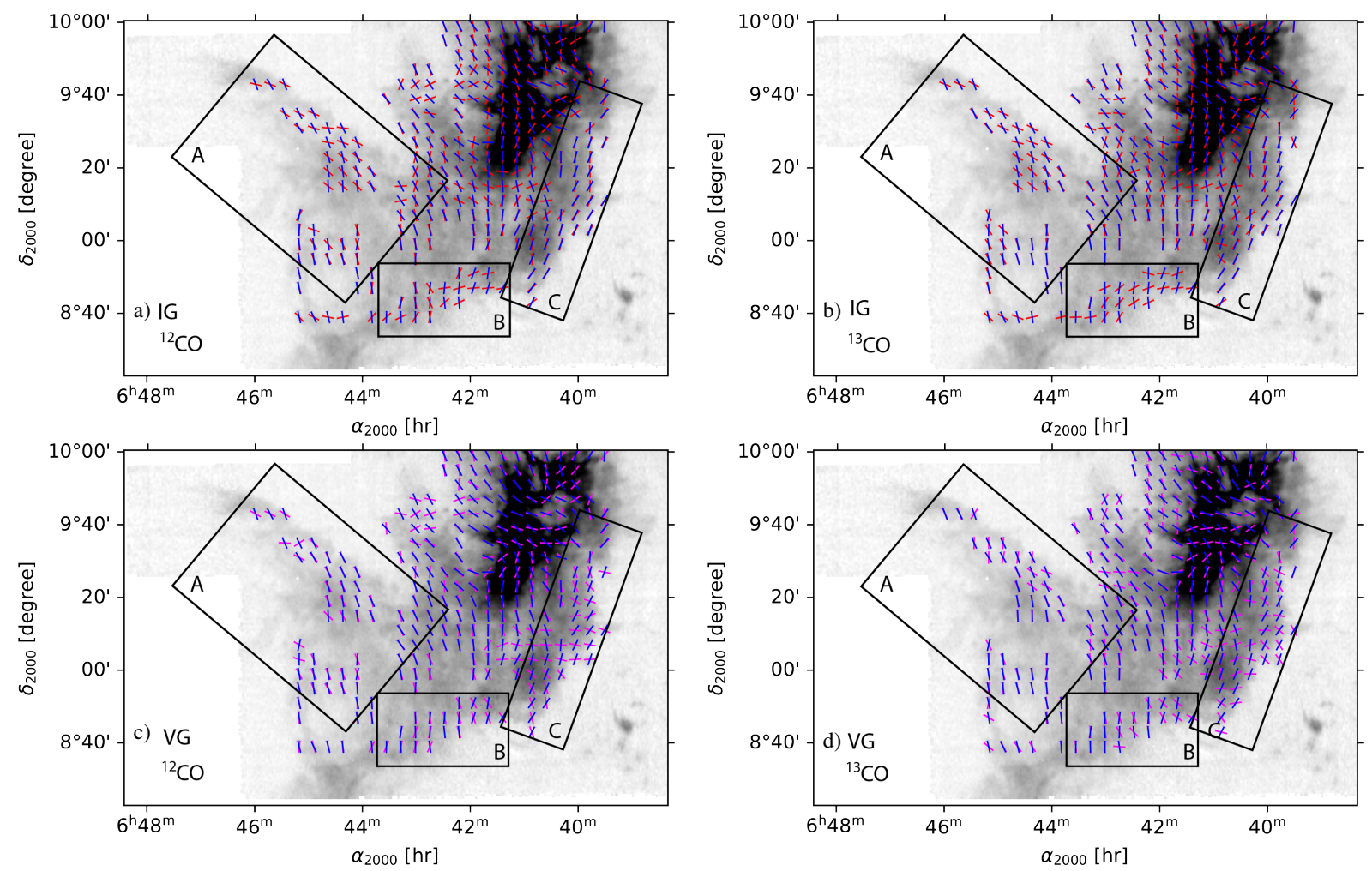

Fig. 10. Southern part of Mon OB 1 east (corresponding to the red box in Fig. 3) with the POS magnetic field orientation represented by the blue segments, overlaid on the TRAO ${ }^{12} \mathrm{CO}$ integrated intensity gray-scale map. Top row: red segments show the orientation of the rotated intensity gradients based on the ${ }^{12} \mathrm{CO}$ and ${ }^{13} \mathrm{CO}$ tracers (panels $a$ and $b$ respectively). Bottom row: magenta segments show the orientation of the rotated velocity gradients based on the ${ }^{12} \mathrm{CO}$ and ${ }^{13} \mathrm{CO}$ tracers ( $c$ and $d$ respectively).
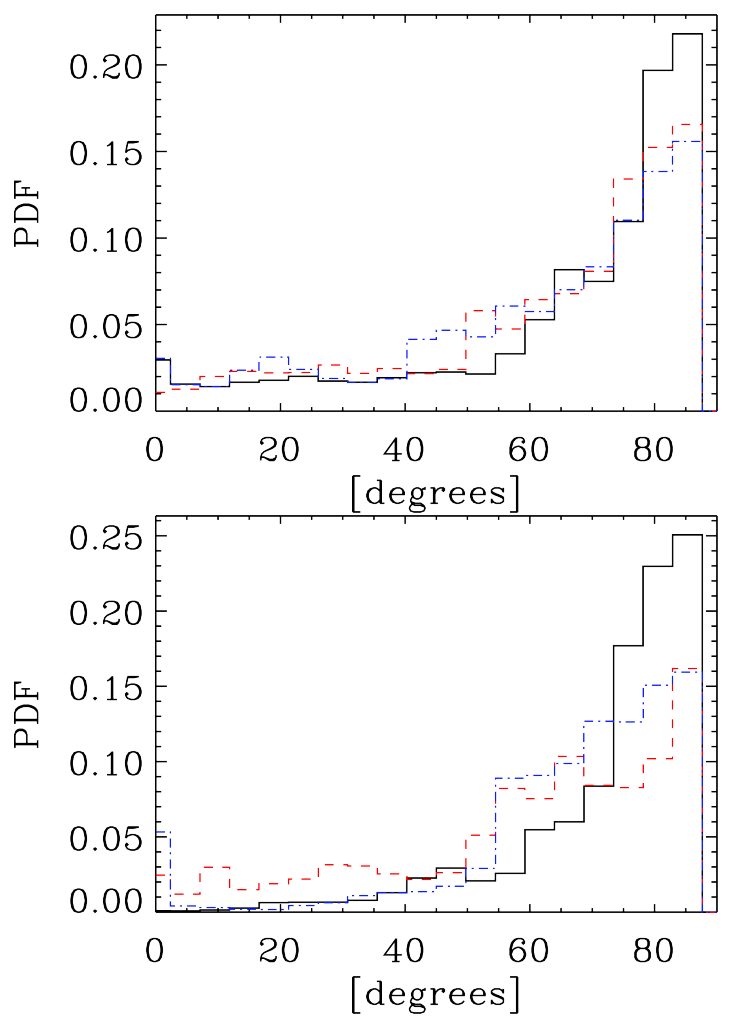

Fig. 11. PDFs of the absolute differences between the POS magnetic field derived from the Planck data and the IGs in black (plain curve), VGs in red (dashed curve), VChGs in blue (dash-dotted curve) for the northern part. Top: based on ${ }^{12} \mathrm{CO}$ data. Bottom: based on ${ }^{13} \mathrm{CO}$ data.
Fig. 11). However, the global behavior is very similar between the VGs and the VChGs. For this reason, we chose not to add the corresponding plots.

\subsubsection{Southern part}

The magnetic field traced by interstellar dust emission polarization in the southern part of the cloud seems to have two major parts. A uniform magnetic field orientated north-east to southwest extends from the eastern edge of the cloud to the densest part, even in regions with no significant $\mathrm{CO}$ detections, and is roughly perpendicular to the Galactic plane (see left panel of Fig. 3). Figure 12 shows the first moment map of ${ }^{12} \mathrm{CO}$ emission, in which we observe that an elongated structure that joins the main cloud at the eastern border and follows this magnetic field direction, is detected at a $v_{\mathrm{lsr}}$ from 8 to $8.4 \mathrm{~km} \mathrm{~s}^{-1}$ in both ${ }^{12} \mathrm{CO}$ and ${ }^{13} \mathrm{CO}$, whereas the rest of the cloud has velocities in the range $3-7 \mathrm{~km} \mathrm{~s}^{-1}$. In the southernmost part, the Planck magnetic field is orientated south-north.

Figure 10 shows the POS magnetic field from the Planck data and the rotated velocity and intensity gradients, to illustrate the derived local magnetic field direction. Contrary to the northern part, the directions of the velocity and intensity gradients differ in more extended regions. We schematically define three regions (rectangles A, B, and C in Fig. 10) to identify the special cases of the relative orientation between the Planck magnetic field and the gradients rotated by $90^{\circ}$, one-by-one. We note that GonzálezCasanova \& Lazarian (2017) found the VGs to be a more robust tool than the IGs, as they are stable to different regimes (subsonic or supersonic). We observe a better agreement between the Planck magnetic field and the VG magnetic field than the 


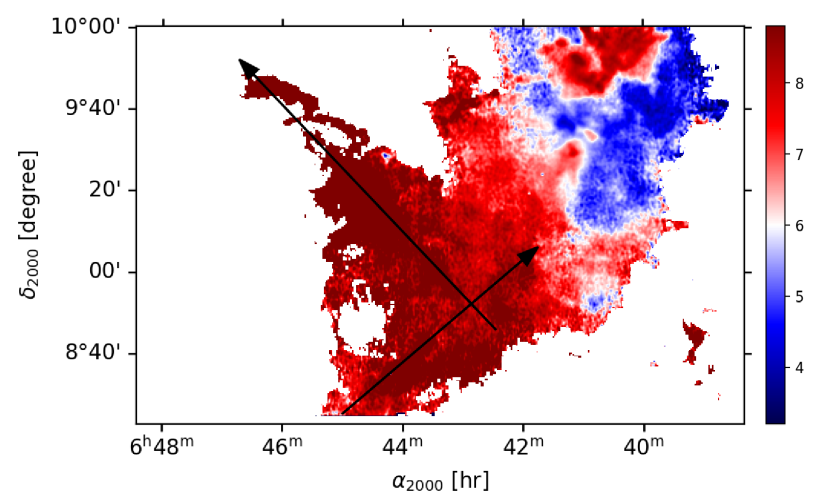

Fig. 12. First moment map of ${ }^{12} \mathrm{CO}$ emission, in $\mathrm{km} \mathrm{s}^{-1}$. The arrows show the cuts used for the position-velocity diagrams of Fig. 14.

IG magnetic field in rectangle A and NGC 2264 for both gas tracers. In addition, the alignment is tighter in ${ }^{12} \mathrm{CO}$ than in ${ }^{13} \mathrm{CO}$. This is expected because ${ }^{12} \mathrm{CO}$ traces the more extended envelope, which might contribute more to the polarized emission detected by Planck in that LOS. In the southernmost part (rectangle B), VGs and IGs are almost perpendicular to each other, and the magnetic field derived from the VGs agrees with the Planck magnetic field direction, which indicates a candidate shock region. Interestingly, at the western edge (rectangle C), the magnetic field derived from the IGs is aligned with the Planck magnetic field while it is perpendicular to the rotated VGs. This does not correspond to any of the three dynamical cases and this might be attributed to the VGs and IGs being dominated by different regions in the LOS.

The histograms of the absolute difference between the orientation of the magnetic field derived from the polarimetric data and the orientation of the IGs and VGs are reported in Fig. C.4. The distributions also peak at $90^{\circ}$ with a spread that is larger than in the northern part.

We present in the upper panels of Fig. 13 the three cases: quiescent (case i) in blue, candidate collapsing (case ii) in red, and candidate shock (case iii) in yellow. The lower panels show the results when using VChGs instead of VGs. The IGs and VGs globally show the same magnetic field orientation as the polarimetric data. There are signs of collapse around NGC 2264 and at the south-western edge. The ${ }^{12} \mathrm{CO}$ VGs indicate shock-like dynamics near the densest part of the cloud. However, it disappears when considering the velocity channel gradients (VChGs). This probably means that several structures are present along the LOS and a combination of higher resolution polarimetric and spectroscopic data would be necessary. Other candidate shock regions are detected in the southern part and at the outer end of the elongated eastern structure, both in VGs and VChGs.

\section{Discussion}

This work aims to study the large-scale magnetic field of the Monoceros OB 1 east cloud and analyze its connection with the cloud's dynamics. For this purpose, Planck polarimetric data and gradients of TRAO spectroscopic measurements are combined. The resolution of the Planck data and the sub-block averaging of the IGs and VGs give access to scales larger than a parsec or larger than a few arcminutes in angular size. This raises questions about whether sub-resolution processes could impact our analysis, and how the data obtained with the large-scale, lowdensity tracer ${ }^{12} \mathrm{CO}$ and the smaller scale, higher density tracer ${ }^{13} \mathrm{CO}$ can be combined to characterize the large-scale magnetic field in this region. We first address these two questions and then discuss the large-scale magnetic field separately in the northern and southern parts of the molecular complex.

The analysis of intensity (or column density) gradients of spectroscopic data for magnetic field studies within MHD turbulence has been applied in sttudies by different authors (Soler \& Hennebelle 2017; Chen et al. 2016; Heyer et al. 2020). In the last sub-section, we discuss our results based on the developments of $\mathrm{Hu}$ et al. (2020) within the interpretations of the column density gradients behavior in the presence of magnetic fields and self-gravity proposed in the literature.

\subsection{Effect of outflows on our large-scale study}

NGC 2264 is actively forming stars which is accompanied by outflows. The outflow activity affects the shape of the emission lines and may affect the calculation of the gradients. Buckle et al. (2012) analyzed the ${ }^{12} \mathrm{CO}(J=3-2)$ emission and $\mathrm{H}_{2}$ emission in the cluster and identified 46 outflows with angular sizes ranging from a few arcseconds to a few arcminutes in a $1 \mathrm{deg}^{2}$ region. They are clustered in a few groups where they are often seen to spatially overlap, effectively covering a minor area in comparison to the $6 \mathrm{deg}^{2}$ studied here. The authors concluded that the outflow activity does not significantly bring energy and momentum and that is not the dominant source of turbulence even on the scale used in their study. In our analysis, the TRAO beam size (47") is larger than most of the identified outflows and the sub-block averaging of the gradients trims the contribution of the most significant outflows. Thus, the outflows have a negligible effect at the final resolution of the gradients. In addition, the conclusions we draw based on the comparison between the gradients and the polarization data, represented in Fig. 13, are scarcely tied to NGC 2264. Instead, they are related to less dense, extended structures.

\subsection{Toward a cloud tomography}

The sub-millimeter emission of the interstellar dust is optically thin, and the signal contains information gathered all along the LOS. The ${ }^{12} \mathrm{CO}$ emission is generally optically thick in molecular clouds. It mostly traces the outer envelopes and less dense material within the cloud, while ${ }^{13} \mathrm{CO}$ is more optically thin and allows us to examine denser parts. We see this difference in the opacity of the two isotopologues as an advantage. When gradients from ${ }^{12} \mathrm{CO}$ and ${ }^{13} \mathrm{CO}$ both agree with polarimetric data, the magnetic field structure is very probably coherent across different layers, from outer envelopes to inner and relatively denser parts. Conversely, when ${ }^{12} \mathrm{CO}$ and ${ }^{13} \mathrm{CO}$ gradients bring different information, it suggests a complex magnetic field morphology through the thickness of the cloud. A similar idea was proposed by $\mathrm{Hu}$ et al. (2019c) who suggested that multiple gas tracers $\left({ }^{12} \mathrm{CO},{ }^{13} \mathrm{CO}, \mathrm{C}^{18} \mathrm{O}, \mathrm{CS}, \mathrm{HNC}, \mathrm{HCO}^{+}, \mathrm{HCN}\right)$ could be used to make a cloud's tomography. While we only have two gas tracers, the available high S/N Planck data in the region allows us to draw a global picture of the interplay between the molecular cloud and the magnetic field. Hsieh et al. (2019) showed that the VGs could safely be applied to both optically thin and optically thick gas tracers to infer the magnetic field morphology. In the northern part, the IGs and VGs derived from ${ }^{12} \mathrm{CO}$ and ${ }^{13} \mathrm{CO}$ data (Fig. 9) globally provide the same direction of the magnetic field. Since ${ }^{13} \mathrm{CO}$ was found to be essentially optically thin in this region (Montillaud et al. 2019b), this indicates that the largescale magnetic field orientated south-north is permeating the filaments at least in gas layers of volume densities on the order of 

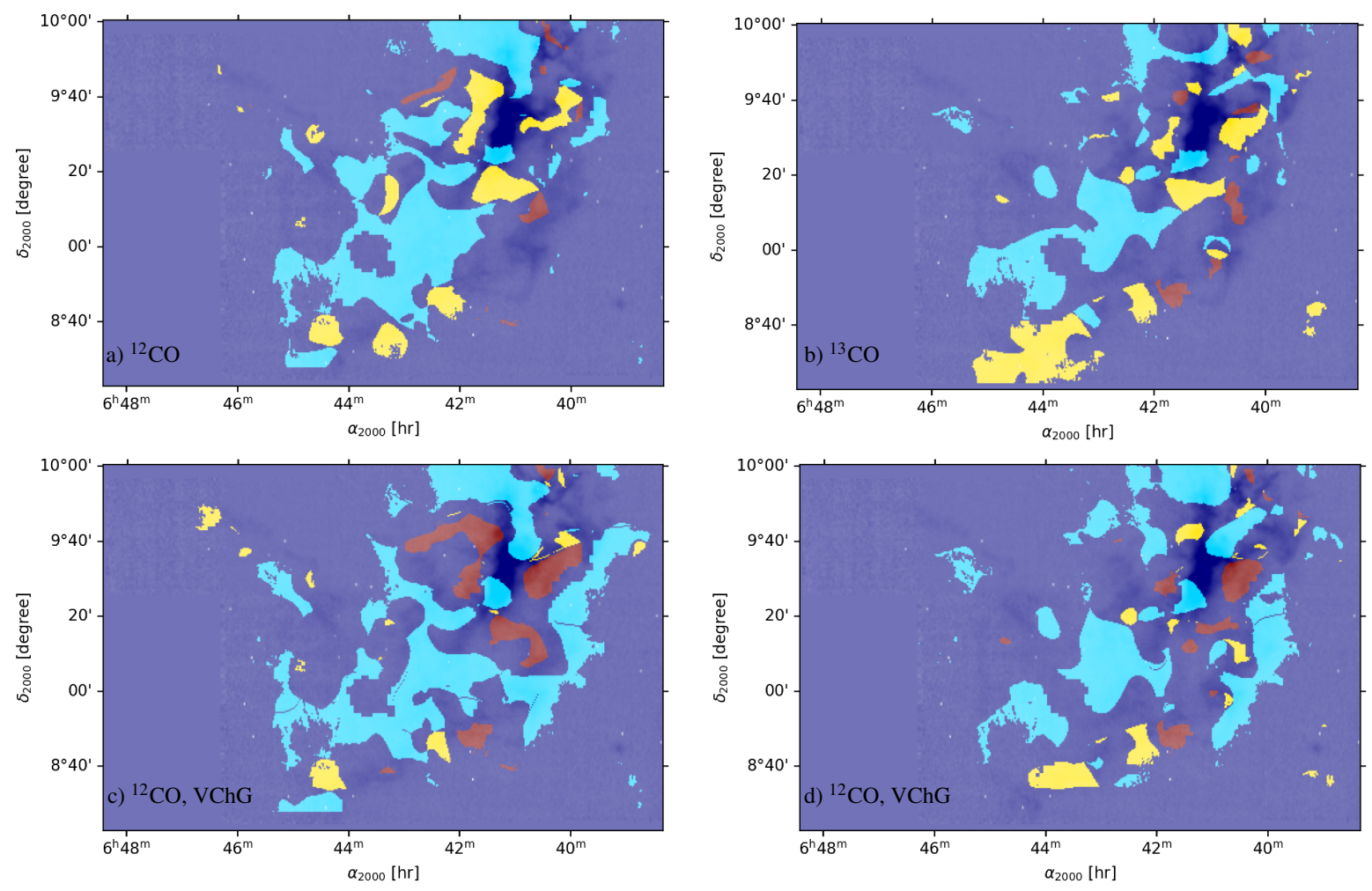

Fig. 13. Southern part of Mon OB 1 east. The blue shaded patterns correspond to the regions where gravity is not dominant (case i), the red shaded patterns correspond to the candidate collapsing regions (case ii), and the yellow shaded patterns correspond to the candidate shock regions (case iii), overlaid on the ${ }^{13} \mathrm{CO}$ integrated intensity grey scale map. Top row: based on the comparison between the velocity gradients, intensity gradients and the POS magnetic field direction derived from Planck: (a) based on ${ }^{12} \mathrm{CO}$ data; $(b)$ based on ${ }^{13} \mathrm{CO}$ data. Bottom row: based on the comparison between the velocity channel gradients, intensity gradients and the POS magnetic field direction derived from Planck: $(c)$ based on ${ }^{12} \mathrm{CO}$ data; $(d)$ based on ${ }^{13} \mathrm{CO}$ data.

those probed by those transitions $\left(\sim 10^{2}-10^{3} \mathrm{~cm}^{-3}\right)$. In the southern part, the magnetic field direction in the cloud's envelope, traced by the IGs and VGs from the ${ }^{12} \mathrm{CO}$ data is different from the magnetic field direction in the denser part of the cloud, traced by ${ }^{13} \mathrm{CO}$ (Fig. 13). In the envelope and the more diffuse gas, the magnetic field is in the south-north and south-east to northwest direction, while in the denser part, it is in the north-east to south-west direction. Rapson et al. (2014) and Park \& Sung (2002) showed that the cloud has a complex, continuous star formation history, with at least one past burst in the southern part of the cloud outside of NGC 2264 and one ongoing inside the cluster. Both studies concluded that the cloud is generally quiescent if not considering NGC 2264. Our analysis supports this statement as we observe a global agreement between the magnetic field directions derived from the spectroscopic gradients and polarimetric data globally in the cloud.

In the two following sections, we discuss each part of the cloud separately.

\subsection{Northern filaments}

The northern part of the cloud seen in submillimeter and radio wavelengths has a filamentary shape that bifurcates into two filaments. Montillaud et al. (2019b) identified the structures as the north-main, north-western, north-eastern filaments, and the junction region (Fig. 4) and showed that the north-western and north-eastern filaments are moving towards each other, suggesting that they are experiencing a collision at their junction. We first re-examine this scenario using our results and then discuss the contribution of the magnetic field to the stability of the cloud substructures.
Our results suggest that the north-main and north-eastern filaments originally formed one structure. The following findings support this idea. First, the north-main and north-eastern filaments have similar polarized intensities properties: their $Q$ and $U$ parameters shown in Fig. 5 have globally the same polarities, negative and positive respectively, while the north-western filament has an opposite polarity, with both $Q$ and $U$ being negative. Second, they exhibit similar behaviors in intensity and velocity gradient orientations with respect to the observed POS magnetic field: the IGs and VGs both agree with the polarimetric measurements regarding the derived magnetic field orientation. The difference in the angles in the north-main and north-eastern filaments, shown in the panels $\mathrm{b}$ and $\mathrm{c}$ of Fig. 9, do not exceed $30^{\circ}$. This is true to a greater extent if we consider the northern part of the north-eastern filament, where polarimetric and spectroscopic data show the same orientation of the magnetic field in both ${ }^{12} \mathrm{CO}$ and ${ }^{13} \mathrm{CO}$.

We also detected a signature of a shock in the diffuse gas using the comparison of the IGs and VGs of ${ }^{12} \mathrm{CO}$ with the polarimetric data. It is located in the northernmost end of the cloud between the two northern filaments. The ${ }^{13} \mathrm{CO}$ data does not show such a trend. This may either mean that the dense structures were formed before the collision or indicate that the dissipation of the shock in the diffuse gas takes longer time. The shock detection in the combination of polarimetric and spectroscopic data supports the hypothesis of a collision of the filaments proposed by Montillaud et al. (2019b).

Our results shed new light on the findings of Montillaud et al. (2019b) who interpreted the north-main and north-eastern filaments as two structures that were originally distinct and are in 
the process of merging at the level of the junction region, while the north-western filament was understood as the continuity of the north-main filament. Our analysis suggests that the northmain and north-eastern filaments were one and entered into a collision with the north-western filament. At the same time, the magnetic field has been dragged along with the matter during the evolution of the structures.

\section{Magnetic support and filament stability}

The northern filaments have a large population of dense cores (Montillaud et al. 2019a; Rapson et al. 2014). An analysis by Montillaud et al. (2019b), based on the Herschel data, suggested that the filaments are subject to fragmentation if the magnetic field does not provide support. In Sect. 4.2, we used the DCF method to estimate the strength of the magnetic field in the northern filaments. We adopted an approach proposed by Houde et al. (2009) to account for beam dilution. However, the DCF method, even with the applied corrections, can be uncertain by a factor of two or more, as discussed in Crutcher (2012). Also, there exists an uncertainty on the determination of $n_{\mathrm{H}_{2}}$ from observations of ${ }^{13} \mathrm{CO}$, which is generally assumed to be a tracer of gas at $n=10^{3} \mathrm{~cm}^{-3}$ rather than the values adopted here $\left(n=10^{2} \mathrm{~cm}^{-3}\right)$, supported by recent studies (Evans et al. 2020). With these caveats, it is clear that the absolute value of the B field remains quite uncertain. However, assuming that the errors committed on the magnetic field strength measurements are similar in the various regions, differences between their values may reflect genuine variations from region to region. In the following, we discuss the differences between the regions in the frame of this assumption. Although the magnetic field strength values are similar (below $10 \mu \mathrm{G}$ ) in the north-eastern and north-western filaments, the ratio of the turbulent-to-ordered component is larger for the north-western filament. The ratio between the actual and critical mass to magnetic flux ratio $\lambda$ for the north-western filament $(\lambda \simeq 2.55)$ is about two times larger than for the north-eastern filament $(\lambda \simeq 1.4)$. In contrast, those of the north-main and north-eastern filaments have fewer discrepancies $(\lambda \simeq 0.95)$. This confirms again that the north-western filament is dynamically more active, Furthermore, the magnetic field properties of the north-main and north-eastern filaments are similar with low $\lambda$. We note that if a larger (e.g., by one order of magnitude) gas volume density was taken in the calculation, the strength of the magnetic field would be larger by a factor of $\simeq 3$ and the two northern filaments would be considered as sub-critical (with $\lambda=0.8$, and 0.4 for the north-western and north-eastern), supported by the magnetic field. In addition, we do not detect signs of gravitational accretion, at the considered scales, when comparing the VGs and IGs to the polarimetric data. This indicates that stability against gravity is provided by turbulence or turbulence and magnetic fields. This is in line with other studies that have aimed to explain the inefficiency of star formation in molecular clouds (Zuckerman \& Evans 1974). Simulations (Clark \& Bonnell 2004; Dobbs et al. 2011; Bonnell et al. 2011) and observations (Barnes et al. 2016; Nguyen-Luong et al. 2016) have shown that globally molecular clouds are gravitationally unbound entities with sites of very dense bound gas in which star formation occurs.

The velocity dispersion used in this analysis is provided by the ${ }^{13} \mathrm{CO}$ data, while the polarization data at the given resolution $\left(7^{\prime}\right.$ or $\left.1.5 \mathrm{pc}\right)$ trace the cold dust emission of structures such as cold clumps or dense filaments. Thus, these results characterize the gravitational to magnetic balance at intermediate densities only (lower than $10^{3} \mathrm{~cm}^{-3}$ ) and do not contradict potentially
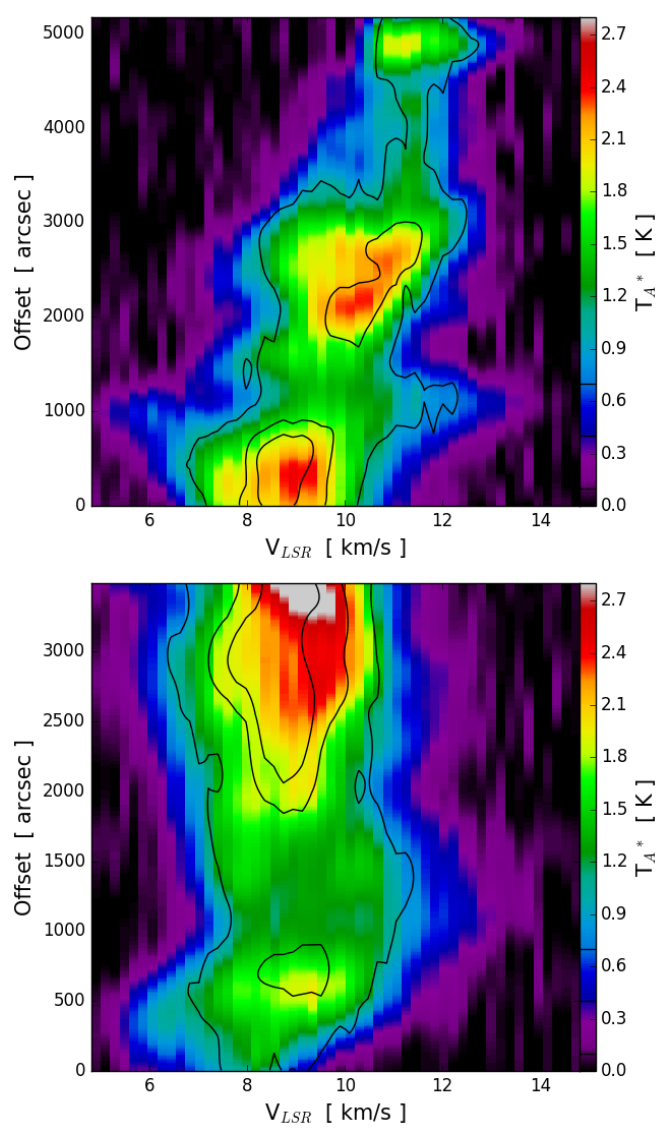

Fig. 14. Position-velocity diagrams of the eastern (top) and southernmost (bottom) inflow candidates in the southern part of the Monoceros OB 1 east. The colorscale shows ${ }^{12} \mathrm{CO}$ emission, while the black lines show contours of ${ }^{13} \mathrm{CO}$ at $T_{\mathrm{A}}^{*}=0.1,0.4$, and $0.7 \mathrm{~K}$. The location of the position-velocity cuts and their orientations are shown by the arrows in Fig. 12.

different behaviors at smaller scales in dense cores. The present study should be complemented with higher angular resolution data to tackle this question. For example, we could consider using the data from the Atacama Cosmology Telescope (ACT, Thornton et al. 2016) at $1^{\prime}$ resolution (Naess et al. 2020) to make the comparison between polarimetry- and spectroscopy-derived orientations of the POS magnetic field but also to estimate the strength using techniques involving polarization-gradient relation within ideal MHD framework from the polarimetric data only (Koch et al. 2012).

\subsection{Southern part}

A large ordered magnetic field orientated roughly perpendicular to the Galactic plane permeates the southern part of the cloud across its densest part, and the whole region seems elongated perpendicular to this magnetic field. We discuss here two structures of interest in this region. The diffuse region framed by rectangle $\mathrm{A}$ in Fig. 10 is elongated alongside its POS magnetic field lines and presents a strong velocity gradient towards the bulk of the cloud (Fig. 14). This suggests an inflow, either channeled by the magnetic field or dragging it. However, in this region, Fig. 13 shows mostly blue patterns (case (i)), where gravity does not dominate the dynamics, and a few small and scattered yellow patches (shocks, case (iii)) - leaving the reason for this possible inflow unclear. Its orientation, which is nearly perpendicular to the Galactic plane, may indicate that the gas follows the Galactic gravitational potential. 
A second structure, at the southernmost end part of the cloud, shown below the rectangles $\mathrm{A}$ and $\mathrm{B}$, covers an extensive velocity range. It appears as a compact red patch in Fig. $12\left(\sim 8 \mathrm{~km} \mathrm{~s}^{-1}\right)$, but its position-velocity profile (Fig. 14) shows that it spans velocities from $\sim 6$ to $12 \mathrm{~km} \mathrm{~s}^{-1}$ over only $\sim 10^{\prime}$, suggesting that it corresponds to a structure greatly inclined with respect to the plane of the sky. Interestingly, the four frames of Fig. 13 show a large yellow pattern indicating a large zone of shock between this structure and the bulk of the cloud, suggesting that this second structure is also flowing into the cloud. This draws a picture where star formation in the main cloud is fueled by inflows of diffuse gas on scales as large as $10-15 \mathrm{pc}$.

We notice other intriguing observations in this region. When using the velocity centroid maps, signs of shocks are present in NGC 2264, but they disappear when using velocity channel maps. Also, the densest area of the southern part, corresponding to the location of the NGC 2264 open cluster, is located precisely at the confluence of two distinct magnetic field regions. These observations would call for further investigation, but they are less relevant for the present study, which is focused on the large-scale magnetic field. Thus, we reserve them for a future study that is more focused on the area of the cluster.

\subsection{Comparison to alternative approaches}

Predictions of the magnetically influenced filament formation and evolution from numerical simulations as well as the corresponding observational pieces of evidence have boosted theoretical investigations of the underlying physical processes. This work employs the technique of intensity and velocity gradients (Yuen \& Lazarian 2017a; Lazarian \& Yuen 2018b; Hu et al. 2018). There are also alternative approaches that utilize gradients of column density to consider. Specifically, Chen et al. (2016) and Soler \& Hennebelle (2017) used the gradients of column density to study its relative orientation with respect to the magnetic field.

It is worth noting that the histograms of relative orientation (HRO) employed in Chen et al. (2016) and Soler \& Hennebelle (2017) studies the relative orientation of the gradients of column density with respect to the magnetic field as revealed by polarization. In contrast, the intensity and velocity gradients techniques effectively allow us to obtain magnetic field direction. Moreover, the VGT and IGT can detect self-gravity effects without employing any polarization data. Hu et al. (2020) showed that the results of identification of collapsing regions obtained without polarization measurements are consistent with the HRO results obtained with polarization measurements. More recently, using velocity gradients, the regions of gravitation collapse were identified in Serpens G3-G6 molecular cloud (Hu et al. 2021). Our present results for the Monoceros OB 1 east also show the consistency of the velocity gradients and HRO predictions. Moreover, the combination of the velocity and intensity gradients of molecular line observations with polarimetric observations of interstellar dust allows us to investigate a possibility of other dynamical processes such as the shock or regions dominated by turbulence and magnetic field that are beyond the techniques utilizing the column density gradients versus the polarization data.

\section{Conclusion and perspectives}

We analyzed the large-scale magnetic field structure of the Monoceros OB 1 east molecular cloud with its complex morphology of interconnected filaments in order to study the possible influence of the magnetic field on the evolution and formation of the cloud and its active star formation. We used the Planck $353 \mathrm{GHz}$ polarized channel to trace the plane-of-sky (POS) component of the magnetic field associated with the cold dust and the novel technique of estimation of intensity and velocity gradients (IG and VG). This approach allowed us to trace dynamically active regions such as shocks or turbulence and magnetic field-dominated regions via a comparison of the polarimetric observations with the spectroscopic data.

The magnetic field in the Monoceros OB 1 east cloud has a more significant influence on the dynamics in the southern part of the cloud than in the northern part. In the northern part, the magnetic field follows the filamentary structure of the cloud in the south-north direction, and this regular field seems to have the same direction in the envelope, as traced by ${ }^{12} \mathrm{CO}$ emission, and in denser parts, as traced by ${ }^{13} \mathrm{CO}$ emission. Our study suggests that the north-main and north-eastern filament were once one whole structure that collided with the north-western filamentary cloud. In this scenario, the magnetic field was kept dragged during the evolution of the cloud, and is probably providing support against fragmentation at the scales larger than a parsec, at least in the north-main filament, in the direction, perpendicular to the magnetic field and the filament. It might also channel a flow of matter along the filament, but we do not detect signs of accretion in the northern filaments at large scales. However, the comparison of unsmoothed gradients and polarization data show small-feature collapsing regions. We conclude that the magnetic fields and turbulence might be the two stabilizing factors in the northern part, at scales larger than a parsec. The southern part, in contrast, shows a complex structure of the magnetic field. The data suggest an inflow of matter towards the dense parts of the cloud, which is channeled along the magnetic field lines in the north-east to south-west direction. Alternatively, the magnetic field could be dragged by the gas motion towards the main cloud. In more diffuse parts, the magnetic field is also generally orientated in the south-north direction.

Our study of Monoceros OB 1 east molecular cloud supports the emerging paradigm: the molecular cloud filaments with higher densities than their environments are mostly elongated perpendicular to the magnetic field, as we see in the southern part. The lower density-contrasted filaments tend to align with the magnetic field, as we observe in the northern part or the diffuse inflow of the southern part.

The studies of the relative orientation between filamentary molecular clouds and magnetic fields indicate the change from parallel to perpendicular with increasing column density (Planck Collaboration Int. XXXV 2016; Soler 2019). In addition, the relative orientation also depends on the characteristics of the environment and the scale such as those of cores or clumps (Zhang et al. 2014; Hull et al. 2017; Alina et al. 2019). Recently Pillai et al. (2020) reported a discovery of a change of the relative orientation from perpendicular to parallel with increasing density in the open cluster in the Serpens south cloud using Sofia HAWC+ data with sub-arcminute resolution. This is a step forward in confirming the paradigm of the relative orientation between matter and magnetic fields in molecular clouds. Further analyses of the Monoceros OB 1 east cloud and the NGC2264 open cluster is of interest to investigate whether this trend can be observed in different molecular clouds.

A detailed analysis of higher S/N data in velocity channels region by region, using higher angular resolution data, would improve our knowledge of the magnetic field properties in the cloud. For example, Naess et al. (2020) obtained an extensive dataset of polarized emission at 90, 150, and $220 \mathrm{GHz}$ from ACT. They developed an algorithm to combine Planck and 
ACT data. Their data include the Monoceros OB 1 east cloud region, and a detailed comparison with the TRAO data might bring new information regarding the large-scale magnetic field. It might also be of interest to derive the strength of the magnetic field using other techniques such as the polarzation-intensity gradient-based method by Koch et al. (2012). Also, an analysis of spectroscopic data of dense gas tracers, such as $\mathrm{N}_{2} \mathrm{H}^{+}$, using the VGs and IGs techniques along with higher resolution polarimetric measurements would be necessary to investigate a possible connection between the magnetic field and the dynamics. This will be especially relevant in complex structures such as the junction region or the NGC 2264 region.

Acknowledgements. This research is funded by the Science Committee of the Ministry of Education and Science of the Republic of Kazakhstan, Grant No. AP08855858, as a part of the project "Study of the polarised emission of the interstellar dust in the Magellanic Clouds using big data and machine learning". D.A. acknowledges the Nazarbayev University Faculty Development Competitive Research Grant Programme No110119FD4503. E.A. acknowledges support from the MES RK grant AP05135753 and the MES RK funded grant "Center of Excellence for Fundamental and Applied Physics" No BR05236454. A.L. and Y.H. acknowledge the support from the NSF AST 1816234, NASA TCAN 144AAG1967 and NASA ATP AAH7546. Flatiron Institute is supported by the Simons Foundation. This work was supported by the Programme Nationa "Physique et Chimie du Milieu Interstellaire" (PCMI) of CNRS/INSU with INC/INP co-funded by CEA and CNES. T.L. acknowledges the supports from the international partnership program of the Chinese academy of sciences through grant no. 114231KYSB20200009, National Natural Science Foundation of China (NSFC) through grant NSFC no.12073061, and Shanghai Pujiang Program 20PJ1415500. This research used JPBLib, an IDL library developed by JeanPhilippe Bernard, and Astropy, a community-developed core Python package for Astronomy.

\section{References}

Alina, D., Montier, L., Ristorcelli, I., et al. 2016, A\&A, 595, A57

Alina, D., Ristorcelli, I., Montier, L., et al. 2019, MNRAS, 485, 2825

André, P., Di Francesco, J., Ward-Thompson, D., et al. 2014, Protostars and Planets VI, 27

Ballesteros-Paredes, J., Klessen, R. S., Mac Low, M. M., \& Vazquez-Semadeni, E. 2007, in Protostars and Planets V, eds. B. Reipurth, D. Jewitt, \& K. Keil, 63

Barnes, P. J., Hernandez, A. K., O’Dougherty, S. N., et al. 2016, ApJ, 831, 67

Beckwith, S. V. W., Sargent, A. I., Chini, R. S., \& Guesten, R. 1990, AJ, 99, 924

Beresnyak, A., \& Lazarian, A. 2019, Turbulence in Magnetohydrodynamics

Bond, J. R., Kofman, L., \& Pogosyan, D. 1996, Nature, 380, 603

Bonnell, I. A., Smith, R. J., Clark, P. C., \& Bate, M. R. 2011, MNRAS, 410, 2339

Buckle, J. V., Richer, J. S., \& Davis, C. J. 2012, MNRAS, 423, 1127

Cantat-Gaudin, T., Jordi, C., Vallenari, A., et al. 2018, A\&A, 618, A93

Carrière, J. S., Montier, L., Ristorcelli, I., \& Ferrière, K. 2019, in SF2A-2019. Proceedings of the Annual meeting of the French Society of Astronomy and Astrophysics, eds. P. Di Matteo, O. Creevey, A. Crida, G. Kordopatis, J. Malzac, J. B. Marquette, M. N'Diaye, \& O. Venot, Di

Chandrasekhar, S., \& Fermi, E. 1953, ApJ, 118, 113

Chen, W. P., Lee, H. T., \& Sanchawala, K. 2007, in IAU Symp., 237, Triggered Star Formation in a Turbulent ISM, eds. B. G. Elmegreen, \& J. Palous, 278

Chen, C.-Y., King, P. K., \& Li, Z.-Y. 2016, ApJ, 829, 84

Cho, J., \& Vishniac, E. T. 2000, ApJ, 539, 273

Cho, J., Lazarian, A., \& Vishniac, E. T. 2002, ApJ, 564, 291

Clark, P. C., \& Bonnell, I. A. 2004, MNRAS, 347, L36

Clark, S., Peek, J., \& Putman, M. 2014, ApJ, 789, 82

Crutcher, R. M. 2004, in The Magnetized Interstellar Medium, eds. B. Uyaniker, W. Reich, \& R. Wielebinski, 123

Crutcher, R. 2012, ARA\&A, 50, 29

Crutcher, R. M., Wandelt, B., Heiles, C., Falgarone, E., \& Troland, T. H. 2010, ApJ, 725, 466

Dahm, S. E., \& Simon, T. 2005, AJ, 129, 829

Davis, Leverett, J. 1951, Phys. Rev., 81, 890

Dobbs, C. L., Burkert, A., \& Pringle, J. E. 2011, MNRAS, 413, 2935
Doi, Y., Hasegawa, T., Furuya, R. S., et al. 2020, ApJ, 899, 28

Dotson, J. L., Vaillancourt, J. E., Kirby, L., et al. 2010, ApJS, 186, 406

Evans, Neal J., I., Kim, K.-T., Wu, J., et al. 2020, ApJ, 894, 103

Federrath, C. 2016, MNRAS, 457, 375

Fissel, L. M., Ade, P. A. R., Angilè, F. E., et al. 2019, ApJ, 878, 110

Goldreich, P., \& Sridhar, S. 1995, ApJ, 438, 763

González-Casanova, D. F., \& Lazarian, A. 2017, ApJ, 835, 41

Green, G. M., Schlafly, E., Zucker, C., Speagle, J. S., \& Finkbeiner, D. 2019 ApJ, 887, 93

Gregorio-Hetem, J. 2003, Bull. Astron. Soc. Brazil, 23, 13

Hacar, A., Tafalla, M., Kauffmann, J., \& Kovács, A. 2013, A\&A, 554, A55

Heiles, C., \& Crutcher, R. 2005, Magnetic Fields in Diffuse HI and Molecular Clouds, 664 (Springer), 137

Hennebelle, P. 2013, A\&A, 556, A153

Heyer, M., Soler, J. D., \& Burkhart, B. 2020, MNRAS, 496, 4546

Hildebrand, R., Kirby, L., Dotson, J., Houde, M., \& Vaillancourt, J. 2009, ApJ 696, 567

Houde, M., Vaillancourt, J. E., Hildebrand, R. H., Chitsazzadeh, S., \& Kirby, L. 2009, ApJ, 706, 1504

Hough, P. 1962, U.S. Patent 3, 069

Hsieh, C.-h., Hu, Y., Lai, S.-P., et al. 2019, ApJ, 873, 16

Hu, Y., Yuen, K. H., \& Lazarian, A. 2018, MNRAS, 480, 1333

Hu, Y., Yuen, K. H., \& Lazarian, A. 2019a, ApJ, 886, 17

Hu, Y., Yuen, K. H., Lazarian, A., et al. 2019b, ApJ, 884, 137

Hu, Y., Yuen, K. H., Lazarian, V., et al. 2019c, Nat. Astron., 3, 776

Hu, Y., Lazarian, A., \& Yuen, K. H. 2020, ApJ, 897, 123

Hu, Y., Lazarian, A., \& Stanimirovic, S. 2021, ApJ, 912, 2

Hull, C. L. H., Mocz, P., Burkhart, B., et al. 2017, ApJ, 842, L9

Jeong, I.-G., Kang, H., Jung, J., et al. 2019, J. Korean Astron. Soc., 52, 227

Koch, P. M., Tang, Y.-W., \& Ho, P. T. P. 2012, ApJ, 747, 79

Kowal, G., Lazarian, A., \& Beresnyak, A. 2007, ApJ, 658, 423

Lazarian, A., \& Pogosyan, D. 2000, ApJ, 537, 720

Lazarian, A., \& Vishniac, E. T. 1999, ApJ, 517, 700

Lazarian, A., \& Yuen, K. H. 2018a, ApJ, 865, 59

Lazarian, A., \& Yuen, K. H. 2018b, ApJ, 853, 96

Lazarian, A., Yuen, K. H., \& Pogosyan, D. 2020, ApJ, submitted, [arXiv:2002.07996]

Li, H.-B., Goodman, A., Sridharan, T. K., et al. 2014, Protostars and Planets VI, 101

Liu, T., Kim, K.-T., Juvela, M., et al. 2018a, ApJS, 234, 28

Liu, T., Li, P. S., Juvela, M., et al. 2018b, ApJ, 859, 151

Malinen, J., Montier, L., Montillaud, J., et al. 2016, MNRAS, 460, 1934

Maron, J., \& Goldreich, P. 2001, ApJ, 554, 1175

Maury, A. J., Wiesemeyer, H., \& Thum, C. 2012, A\&A, 544, A69

Montier, L., Plaszczynski, S., Levrier, F., et al. 2015a, A\&A, 574, A135

Montier, L., Plaszczynski, S., Levrier, F., et al. 2015b, A\&A, 574, A136

Montillaud, J., Juvela, M., Vastel, C., et al. 2019a, A\&A, 631, L1

Montillaud, J., Juvela, M., Vastel, C., et al. 2019b, A\&A, 631, A3

Naess, S., Aiola, S., Austermann, J. E., et al. 2020, JCAP, 12, 046

Nguyen-Luong, Q., Nguyen, H. V. V., Motte, F., et al. 2016, ApJ, 833, 23

Padoan, P., Bally, J., Billawala, Y., Juvela, M., \& Nordlund, A. 1999, ApJ, 525 318

Park, B.-G., \& Sung, H. 2002, AJ, 123, 892

Pillai, T. G. S., Clemens, D. P., Reissl, S., et al. 2020, Nat. Astron., 4, 1195

Planck Collaboration XXVIII. 2015, A\&A, 594, A28

Planck Collaboration I. 2020, A\&A, 641, A1

Planck Collaboration Int. XIX. 2015, A\&A, 576, A104

Planck Collaboration Int. XXXV. 2016, A\&A, 586, A138

Quinn, J. 2012, A\&A, 538, A65

Rapson, V. A., Pipher, J. L., Gutermuth, R. A., et al. 2014, ApJ, 794, 124

Serkowski, K. 1958, Acta Astron., 8, 135

Soam, A., Liu, T., Andersson, B. G., et al. 2019, ApJ, 883, 95

Soler, J. D. 2019, A\&A, 629, A96

Soler, J. D., \& Hennebelle, P. 2017, A\&A, 603, A64

Soler, J. D., Hennebelle, P., Martin, P. G., et al. 2013, ApJ, 774, 128

Thornton, R. J., Ade, P. A. R., Aiola, S., et al. 2016, ApJS, 227, 21

Wolf-Chase, G., Moriarty-Schieven, G., Fich, M., \& Barsony, M. 2003, MNRAS, 344,809

Xu, S., Ji, S., \& Lazarian, A. 2019, ApJ, 878, 157

Yuen, K. H., \& Lazarian, A. 2017a, ArXiv e-prints, [ArXiv:1703.03026]

Yuen, K. H., \& Lazarian, A. 2017b, ApJ, 837, L24

Zaldarriaga, M. 1998, ApJ, 503, 1

Zhang, Q., Qiu, K., Girart, J., et al. 2014, PASP, 792, 116

Zuckerman, B., \& Evans, N. J., I. 1974, ApJ, 192, L149 


\section{Appendix A: Relative orientation between the filaments and the magnetic fields}

\section{Filament detection algorithm}

A number of algorithms aimed at the identification of filamentary patterns have led to the discovery of a cosmic web structure among the galaxy clusters (Bond et al. 1996) and of the ubiquitous nature of the interstellar filaments as revealed by Herschel (André et al. 2014). Among the many, we used the Rolling Hough Transform (RHT) (Clark et al. 2014) because it allows us to identify structures regardless of their intensity relative to the maximum intensity over the map, which means that not only the densest filament is detected. Furthermore, it allows us to trace the structure extent, which means that not only the crest is detected. The RHT is based on the Hough Transform (Hough 1962). At each pixel, it centers a user-defined kernel of rectangular shape, computes the total intensity for every position angle in the parameter space and builds a histogram from which a final value is picked up. The method strongly depends on the input parameters, such as kernel size, length, and threshold for the determination of the final angle over the histogram, which are chosen for each map separately depending on the resolution and the angular size of the structures. Also, the rectangular kernel privileges linear structures and the detected pattern can be more linear than the real filament. However, choosing a relatively short aspect ratio for the kernel, that is between two and four, makes it possible to reflect the global curvature of the structure. We applied the RHT to the Planck column density map. The explored parameter ranges were between $15^{\prime}$ and $31^{\prime}$ for the length and $3^{\prime}$ to $9^{\prime}$ for the width, the smoothing kernel is set to be equal to the kernel width. The final parameters are $21^{\prime}$ and $6^{\prime}$ for the kernel's length and width, respectively; and the normalised histogram threshold for the identification of the maximum is set to 0.65 . It is worth noting that for the detection of more ramified structures, as, for example, in the Herschel map (Montillaud et al. 2019a), we would suggest using the optimized version which does not require to set up the histogram threshold (Carrière et al. 2019).

\section{Results}

The RHT was applied to the Planck column density map in order to quantitatively define the two northern filaments as seen in the continuum map and study the variations in the relative orientation of the filaments with respect to the magnetic field. We represent in Fig. A.1 the detected structure in the northern part of the Monoceros OB-1 east cloud, where we clearly observe the three prominent filaments. Figure A.2 shows the PDFs of the magnetic field angles in the north-eastern and northwestern filaments. The red dashed lines represent the attempts of Gaussian fits to the data. In the north-eastern filament the magnetic field angle spans from roughly $-10^{\circ}$ to $15^{\circ}$ with the maximum of the fit at $3^{\circ}$, while in the north-western filament it is almost uniformly distributed between $-10^{\circ}$ and $5^{\circ}$ with a peak at $-2.6^{\circ}$. We determine the average dispersion along each filament as the mean value of the standard deviations of the angles taken perpendicular to the crest. We obtain $5.4^{\circ}$ and $5.3^{\circ}$ for the north-eastern and north-western filaments, respectively. Figure A. 3 shows the PDFs of the absolute difference between the magnetic field angles and the filament orientations. We observe that the magnetic field and the matter are mostly aligned with respect to each other in both filaments.

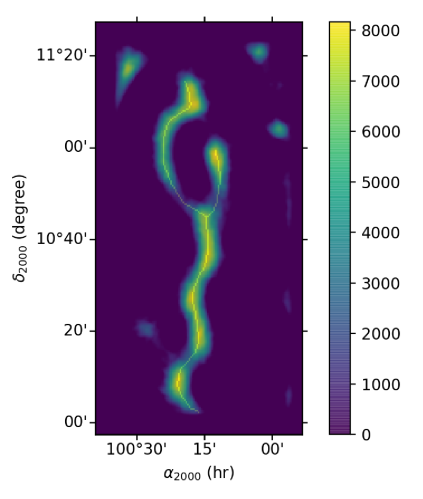

Fig. A.1. Rolling Hough Transform (RHT) intensity calculated over the Planck column density map. The yellow curve shows the crest of the detected structure.
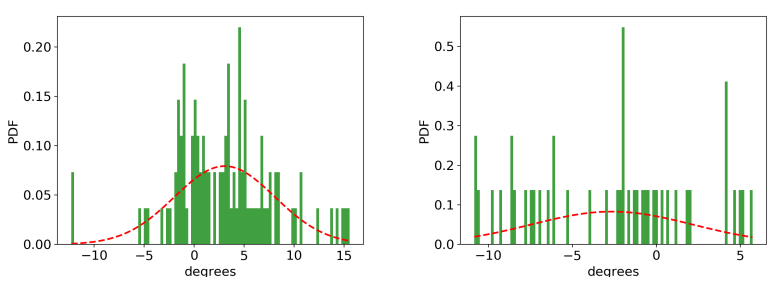

Fig. A.2: PDFs of the POS magnetic field angles derived from the Planck $353 \mathrm{GHz}$ data in the north-eastern (left panel) and the north-western (right panel) filaments detected by the Rolling Hough Transform. The red dashed curve represents the Gaussian fits.
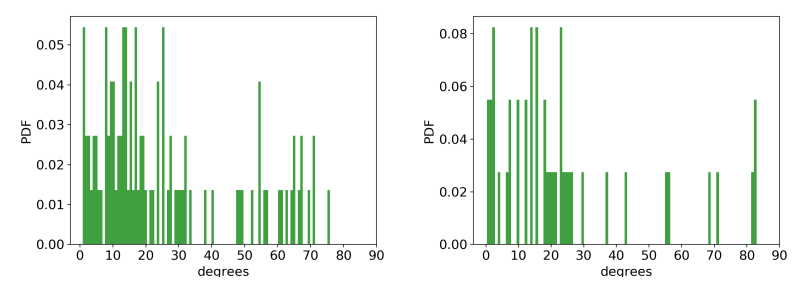

Fig. A.3: PDFs of the absolute difference between the POS magnetic field angles and the orientation of the matter structures derived using the Rolling Hough Transform in the north-eastern (left panel) and the north-western (right panel) filaments.

\section{Appendix B: Determination of the magnetic field strength using angular structure function}

In this section, we briefly summarize the method described in Hildebrand et al. (2009) The two-point polarization-angle structure function is defined as a root mean square of the angle difference between a pair of points located at a distance, $l$, from one another. In terms of the Stokes parameters, the polarizationangle structure function has the following expression (Planck Collaboration Int. XIX 2015; Alina et al. 2016):

$$
\begin{array}{r}
S(l)=\left[\frac { 1 } { N ( l ) } \sum _ { i = 1 } ^ { N ( l ) } \left(\frac { 1 } { 2 } \operatorname { a r c t a n } \left[U(\mathbf{x}) Q\left(\mathbf{x}+\mathbf{l}_{i}\right)-Q(\mathbf{x}) U\left(\mathbf{x}+\mathbf{l}_{i}\right),\right.\right.\right. \\
\left.\left.\left.Q(\mathbf{x}) Q\left(\mathbf{x}+\mathbf{l}_{i}\right)+U(\mathbf{x}) U\left(\mathbf{x}+\mathbf{l}_{i}\right)\right]\right)^{2}\right]^{1 / 2} .
\end{array}
$$

The approach from Hildebrand et al. (2009) was as follows. Within the assumption that the contribution of the large-scale structured magnetic field and the turbulent component of the magnetic field are independent, both contribute quadratically along with the measurement uncertainty $\sigma_{M}$ to the total angle 

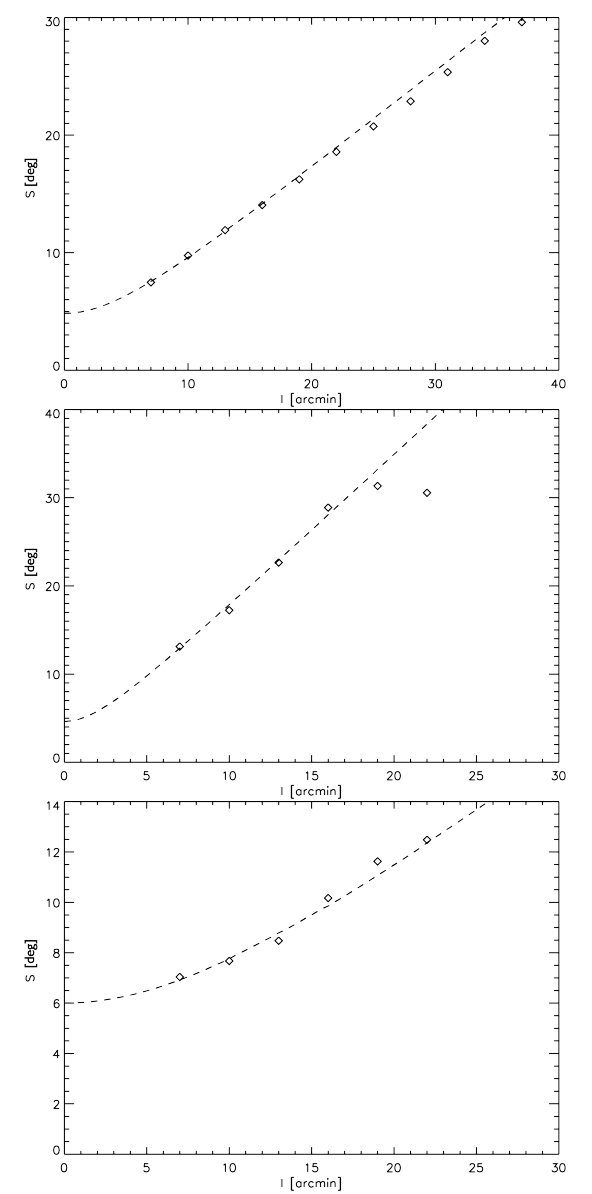

Fig. B.1: Structure function calculated according to Eq. B.1 for the north-main, north-western and north-eastern filaments (diamonds) for different lags $l$ and the fit to the data following Eq. B.2 (dashed curve).

structure function:

$S \simeq \sqrt{b^{2}+m^{2} l^{2}+\sigma_{M}(l)^{2}}$,

where $m l$ defines the large-scale component and $b$ corresponds to the turbulent dispersion of the large-scale magnetic field. In practice, $b$ can be determined from the zero-intercept of the measured polarization angle structure function. This allows us to calculate the large-scale magnetic field component using the DCF method:

$B_{0} \simeq \sqrt{8 \pi \rho} \frac{\sigma(v)}{b}$

We used the same lags for the calculation of $S$ as in Sect. 3.2. The resulting curves and the fits are represented in Fig. B.1. The uncertainties are calculated by propagation of the standard error between the fit and the data.

It is worth noting that Lazarian et al. (2020) proposed the following expression for the structure function of polarization angle to be more appropriate in the frame of the MHD turbulence:

$S F\{V\}(R)=q R^{1+m}+p R^{2}$

where $q$ and $p$ are constants, $V$ is the velocity centroid, and $R$ is the distance separation. In other words, at the scales less than the injection scale of the turbulence, one measures the scaling that reflects the power-law scaling of 3D turbulent motions. Using the representation given by Eq. B.4, Lazarian et al. (2020) proposed their new technique of using the structure functions that may be used in studies of the magnetic fields in molecular clouds.

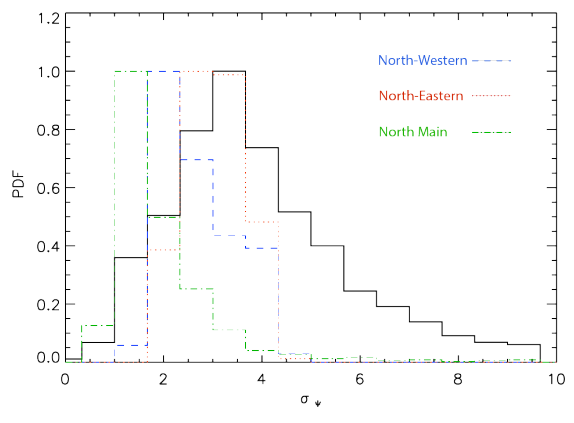

Fig. C.1: PDFs of the uncertainty on polarization angle, $\sigma_{\psi}$, computed using Bayesian analysis (Montier et al. 2015b) inside the area delimited by the contour in Fig. 1 (plain black) and inside the north-main(green dash-dotted line), north-eastern (red dotted line), and north-western (blue dashed line) VCSs.

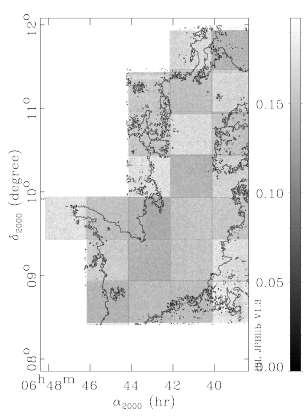

Fig. C.2: Rms noise map of the ${ }^{13} \mathrm{CO}$ data, in $\mathrm{K}$. The contours are given by ${ }^{12} \mathrm{CO}$ integrated intensity at $2 \mathrm{~K} \mathrm{~km} \mathrm{~s}^{-1}$, same as in Fig. 1

\section{Appendix C: Additional figures}

Here we represent:

- the uncertainty on $\psi$ in the area represented by contours in Fig. 1, excluding pixels with $\mathrm{S} / \mathrm{N}(p) \leq 2$ and $\sigma_{\psi}>10^{\circ}$. The $\mathrm{S} / \mathrm{N}(\psi)$ in the north-main, north-eastern and north-western VCSs are shown in green (dash-dotted), red (dotted), and blue (dashed) respectively and generally do not exceed $5^{\circ}$.

- the rms noise map of the TRAO 14 -meter ${ }^{13} \mathrm{CO}$ data in greyscale with the same contours of the ${ }^{12} \mathrm{CO}$ integrated intensity at $2 \mathrm{~K} \mathrm{~km} \mathrm{~s}^{-1}$ as in Fig. 1 .

- the PDFs of the absolute difference between the magnetic field orientation derived from the Planck $353 \mathrm{GHz}$ polarized channel data and the orientation of the IGs and VGs derived from the TRAO 14-meter telescope CO data. The unsmoothed gradients are used to produce the PDF shown in Fig. C.3, while the PDF in Fig. C. 4 is built using the CO data smoothed to the resolution of $7^{\prime}$.

\section{Appendix D: MHD turbulence, reconnection and Gradient Technique}

The Gradient Technique (GT) is rooted in the advanced MHD turbulence theory and the theory of turbulent magnetic reconnection. The Velocity Gradient Technique (VGT) is a branch of the GT and the Intensity Gradient Technique (IGT) can be viewed as an outgrowth of the VGT. Here we briefly explain the basic elements of the theory that are essential for understanding the VGT and IGT. An in-depth discussion of the properties of MHD 

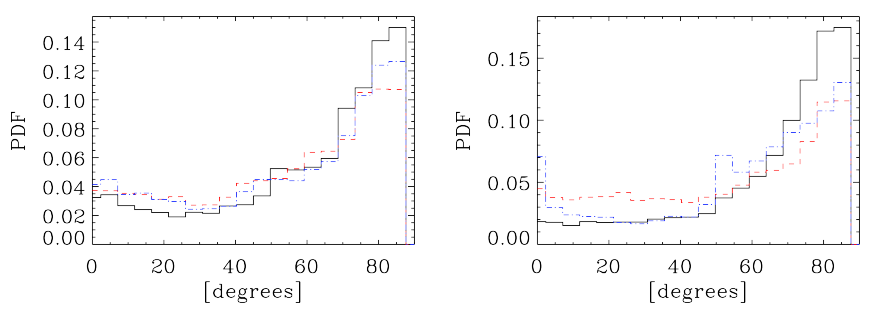

Fig. C.3: PDFs of the absolute differences between the POS magnetic field derived from the Planck data and the unsmoothed ( $47^{\prime}$ resolution) IGs in black (plain curve), VGs in red (dashed curve), VChGs in blue (dash-dotted curve) for the northern part. Left panel: Based on ${ }^{12} \mathrm{CO}$ data. Right panel: Based on ${ }^{13} \mathrm{CO}$ data.
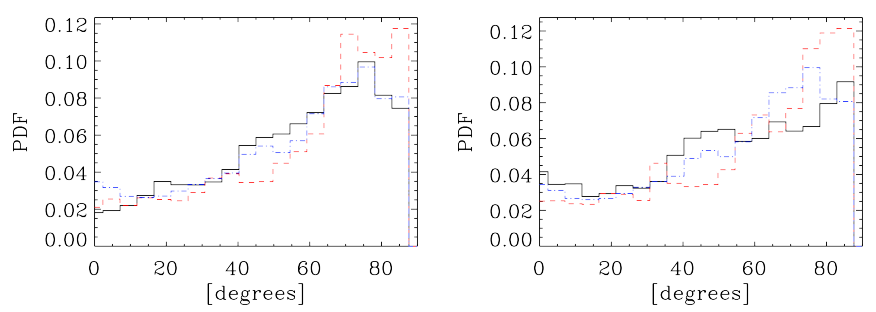

Fig. C.4: PDFs of the absolute differences between the POS magnetic field derived from the Planck data and the IGs in black (plain curve), VGs in red (dashed curve), VChGs in blue (dashdotted curve) for the southern part. Left panel: based on ${ }^{12} \mathrm{CO}$ data. Right panel: based on ${ }^{13} \mathrm{CO}$ data.

turbulence and its relation to the turbulent reconnection can be found in the monograph by Beresnyak \& Lazarian (2019).

The current understanding of MHD turbulence theory is related to the pioneering study in Goldreich \& Sridhar (1995, hereafter GS95). There, the concept of scale-dependent anisotropy increasing with the decrease of the scale was first introduced. However, this GS95 anisotropy scaling is derived in the global magnetic field reference frame, in which the predicted scaling is not observable. In fact, the scale-dependent anisotropy is present in the so-called "local" system of reference. This concept of the local system of reference is frequently a point of confusion for many researchers and it is thus discussed below. Here we would like to stress that for the GT, the notion of the local system of reference is central and crucial.

The local system of reference naturally arises in the theory of turbulence that is based on the turbulent reconnection (Lazarian \& Vishniac 1999, LV99 hereafter). There, it was demonstrated that MHD turbulence can be presented as a collection of eddies with their rotation axis aligned with the magnetic field. This physical picture is possible as the time scale for the turbulent reconnection coincides with the eddy turnover time. The turbulent reconnection is an essential part of the dynamics of turbulent eddies, which enables the mixing of magnetic field lines perpendicular to the magnetic field direction. In this direction, turbulent eddy motions do not experience the magnetic back-reaction. In other words, if an eddy is rotating around its surrounding magnetic field, the eddy evolves in a hydrodynamic type manner. The evolution of this type of eddies with their rotation axis parallel to the magnetic field direction presents the path of the minimal resistance for the energy cascade. Therefore, most of the energy is channeled through such an anisotropic cascade.

The motions perpendicular to the local magnetic field are Kolmogorov types that arise due to the absence of the back- reaction of magnetic field. Therefore the Kolmogorov relations are valid for the eddy size $l_{\perp}$, perpendicular to the magnetic field, and for the eddy perpendicular velocity $v_{l}$, namely, $v_{l} \sim l_{\perp}^{1 / 3}$.

As the eddies mix up the magnetic field, this causes Alfvén waves to propagate along the magnetic field at a velocity, $V_{A}$. The period of the Alfvén wave generated this way $\sim l_{\|} / V_{A}$ is equal to the turnover time of the magnetic eddies, the latter being $\sim l_{\perp} / v_{l}$. The relation $l_{\|} / V_{A} \sim l_{\perp} / v_{l}$ corresponds to the critical balance in GS95 theory. However, in the latter theory, the scales $l_{\perp}$ and $l_{\|}$ are measured with respect to the mean rather than to the local magnetic field direction.

The considerations above reflect the spirit of arguments that was used in LV99 to derive the following relation between the parallel and perpendicular scales of the eddies in the local reference frame of the eddies:

$l_{\|} \simeq L_{i n j}\left(\frac{l_{\perp}}{L_{i n j}}\right)^{\frac{2}{3}} M_{A}^{-4 / 3}$

where $M_{A}$ is the ratio of the injection velocity, $v_{L}$, to the Alfvén speed, $V_{A}$, while $L_{i n j}$ is the injection scale of turbulence. This universal scale-dependent anisotropy of Alfvénic turbulence in the local magnetic field reference frame has been demonstrated in Cho \& Vishniac (2000); Cho et al. (2002), and Maron \& Goldreich (2001).

Combining Eq. D.1 and the "critical balance" expressed in the local reference frame, namely, $l_{\perp} V_{A} \sim l_{\|} v_{l}$, we can get the scaling relation for velocity fluctuations (see Lazarian \& Vishniac 1999):

$v_{l} \simeq v_{L}\left(\frac{l_{\perp}}{L_{i n j}}\right)^{\frac{1}{3}} M_{A}^{\frac{1}{3}}$

where $v_{L}$ is the injection velocity of turbulence.

The eddies parallel to the local direction of the magnetic field present a remarkable possibility of determining the direction of the magnetic field by measuring the gradients of the absolute value of velocity. Indeed, the latter is perpendicular to the local magnetic field direction (see Hu et al. 2020 for pictorial illustration). Thus, the velocity gradients are capable of tracing the magnetic fields. In Alfvénic turbulence, the magnetic field amplitude fluctuations are proportional to the velocity fluctuations. ${ }^{5}$ Therefore, magnetic amplitude gradients can also be used to trace magnetic field direction. It is important to note the velocity gradient's amplitude for Kolmogorov-type eddies to be increasing with the decrease of the eddy size, namely, $v_{l} / l_{\perp} \sim$ $l_{\perp}^{-2 / 3}$. Consequently, the smallest resolved eddies corresponding to the telescope beam size contribute most to the observed gradient measurement. Also, these smallest eddies trace the local magnetic field around them well.

The relation between the turbulent velocity fluctuations and density fluctuations is not so direct as between the velocities and magnetic field (Kowal et al. 2007). However, for a range of parameters, the density acts as a so-called "passive scalar" and is advected by velocity fluctuations. In this case, the statistics of velocity is imprinted on the density statistics, and the application of the gradient technique to tracing magnetic field is justified.

Explicitly, since the anisotropic relation indicates $l_{\perp} \ll l_{\|}$, the velocity gradient, $\nabla v_{l}$, and density gradient, $\nabla \rho_{l}$, are scaled

5 From the mathematical point of view, the symmetry of magnetic and velocity fluctuations in Alfvenic turbulence is evident if the description of MHD turbulence with Elsasser variables is employed (see Beresnyak \& Lazarian 2019)). On the intuitive level, this symmetry is related to the Alfven expression that defines $\delta v_{l} \sim \delta b_{l}$. 
as (Hu et al. 2020):

$\nabla \rho_{l} \propto \frac{\rho_{l}}{l_{\perp}} \simeq \frac{\rho_{0}}{c} F^{-1}(|\hat{k} \cdot \hat{\zeta}|) \nabla v_{l}$

$\nabla v_{l} \propto \frac{v_{l}}{l_{\perp}} \simeq \frac{v_{L}}{L_{i n j}}\left(\frac{l_{\perp}}{L_{i n j}}\right)^{-\frac{2}{3}} M_{A}^{\frac{1}{3}}$

where $\rho_{0}$ is the mean density, $\hat{\zeta}$ is the unit vector for the Alfvénic mode (fast mode or slow mode), $c$ is the propagation speed of the corresponding mode, and $v_{l}$ is the turbulence velocity at scale $l$. In addition, $F^{-1}$ denotes the inverse Fourier transformation. The direction of density gradient and velocity gradient is perpendicular to the local direction of the local magnetic field, and this consideration is at the core of analyses based on the VGT and IGT. 